\title{
EVOLUTION PROBLEMS FOR A CLASS OF DISSIPATIVE MATERIALS*
}

\author{
BY \\ PIERRE-M. SUQUET \\ Université Paris VI
}

\begin{abstract}
This work examines the problems of dynamic and quasi-static evolution for a large class of dissipative materials, including viscoplastic, viscoelastic, and elastic perfectly plastic materials. We show that when the potential of dissipation is regular, the displacement solution is regular; however, in the case of perfect plasticity, where the potential is irregular, the solution can be discontinuous. A suitable framework is used in order to account for these discontinuities. Existence theorems are stated, and the boundary conditions are discussed. The evolution equations encountered in this work are strongly nonlinear but with a monotone time-dependent nonlinearity. A direct method of resolution is proposed, since the known results do not apply in this case.
\end{abstract}

1. Introduction. The evolution problem for elasto-plastic solids has developed remarkably in the last ten years, and has taken advantage of the recent advances in convex analysis. In fact, the first to use this tool were Hill, Prager and Hodge who established the velocity principles based on variational arguments (reported in the survey by Koiter [12]).

More recently, a second step was achieved by Moreau [22] and his school and by Duvaut and Lions for the quasi-static evolution of the stress field in perfectly plastic bodies. Moreau built the geometrical theory of "catching up by a moving convex set", and proved directly the existence (and the uniqueness) of the stress history, while Duvaut and Lions [5] used the constructive theory of partial differential equations, including Galerkin approximations, regularization and penalization to obtain the same result. Both have motivated numerous works on nonlinear evolution equations of monotone type (Brezis [2], Moreau [23]), the model of which is:

Find $\sigma(t) \in K(t)$ such that:

$$
d \sigma / d t+\partial I_{K(t)}(\sigma) \ni f(t), \quad \sigma(0)=\sigma_{0},
$$

where $K(t)$ is a family of moving convex sets in a Hilbert space and $I_{K}$ is the indicator function of the convex set $K$. The above-mentioned works generated three directions of study:

(a) some authors were interested in the evolution problem for the velocity (or displacement) field: Nayroles [25], Johnson [9]. The main difficulty of this problem is the

\footnotetext{
* Received January 25, 1980. The author is indebted to Professors Débordes, Duvaut, Geymonat, Moreau, Murat, Nayroles, Nguyen Quoc Son and Strang for stimulating discussions during the elaboration of this work.
} 
regularity of the strain tensor: this point was avoided by Johnson by means of a weak formulation; however, his result was too weak to obtain full information on the strains. Nayroles gave an existence theorem in a space of functions which are not distributions;

(b) the theory of limit loads and shake-down has been investigated by Debordes [4], Johnson-Mercier [10], Nayroles and the Italian school, who give a mathematical discussion of the classical theorems on these subjects;

(c) a wider class of dissipative materials has been introduced by Halphen and Nguyen Quoc Son [7] and Nguyen Quoc Son [26], using the pseudo-potential assumption of Moreau [21]. This assumption, which has a strong thermodynamic basis, leads to monotone evolution equations.

The present work is interested both in points (a) and (c). We want first to present a coherent mathematical study of standard dissipative materials, without specifying the constitutive law. Then we want to prove the existence of a velocity field history for the quasi-static evolution of a perfectly plastic body, using some regularized models. This part completes the work of Johnson on this subject. Moreover it justifies the numerous regularizations currently used to approximate the perfectly plastic law (such as viscoplasticity, viscoelastic material ...).

We shall also study the dynamic problem which appears to be a formal regularization of the quasi-static problem. If we set $U=(\sigma, \dot{u})$, where $\sigma$ is the stress tensor and $\dot{u}$ the velocity field, the evolution equation is:

$$
\frac{d}{d t}\left(\mathscr{A}_{t} U\right)+\mathscr{B}(t, U) \ni f(t), \quad U(0)=U_{0},
$$

where $\left(\mathscr{L}_{t}\right)_{0 \leq t \leq T}$ is a family of linear coercive and continuous operators and $[\mathscr{B}(t, \cdot)]_{0 \leq t \leq T}$ is a family of monotone nonlinear operators (which are not subdifferentials of convex functions).

Equations of type (1.2) with a time-dependent nonlinearity have been studied by numerous authors after the work of Brezis and Moreau on (1.1): Attouch and Damlamian [1], Kenmochi [11], Crandall and Pazy [3], Martin [16], Watanabe, among others. Some of these authors deal with nonlinear operators which are subdifferentials of convex functionals; we shall see that this is not the case in our problem. Others assume that the domains $D[\mathscr{B}(t, \cdot)]$ are equal or have the same closure; this is not the case here. It seems that no general theorem of existence of a strong solution of (1.2) is available under reasonable assumptions. Hence a result of existence and uniqueness is given in our special case, and is proved by a direct method (semi-discretization in time).

The quasi-static problem (with a regular constitutive law) is formally obtained from the dynamic one by neglecting the inertia terms; it is still of type (1.2), but with a time-dependent constraint. The rigorous treatment is, however, different.

The perfectly plastic body is obtained by approximation by regularized potentials. We get an $L^{1}$ estimate on the strains of the regularized model: when the regularization parameter tends to zero, the strain may accumulate and discontinuities might occur. The displacement (or the velocities) are no longer in a classical Sobolev space $\left[W^{1, p}(\Omega)\right]^{3}$, but in a functional space recently introduced in [18] and [25]:

$$
B D(\Omega)=\left\{u \mid u=\left(u_{i}\right), \quad u_{i} \in L^{1}(\Omega), \quad \varepsilon_{i j}(u) \in M^{1}(\Omega)\right\} .
$$

The introduction of this space of discontinuous functions, analogous to the space $B V(\Omega)$ used in shock theory, agrees with the experimental data where one can see the 
strain accumulate in thin bands, considered as discontinuity surfaces. Using $B D(\Omega)$, Strang, Matthies and Christiansen [20] have investigated the direction (b) (limit analysis): this space appears to be also the right functional framework for the variational principles of limit analysis. Matthies [19] also investigated direction (a).

1. Constitutive laws. The total strain $\varepsilon(u)$ of the material is the sum of an elastic part and a nonelastic part:

$$
\begin{aligned}
& \varepsilon_{i j}(u)=\frac{1}{2}\left(\frac{\partial u_{i}}{\partial x_{j}}+\frac{\partial u_{j}}{\partial x_{i}}\right), \\
& \varepsilon_{i j}(u)=\varepsilon_{i j}^{e}+\varepsilon_{i j}^{a n}, \quad \varepsilon_{i j}^{e}(x, t)=A_{i j k h}(x, t) \sigma_{k h}(x, t) .
\end{aligned}
$$

$A, \sigma$, and $u$ denote the elastic compliance coefficients, the stress tensor field, and the displacement field of the material.

We assume that there exists a pseudo-potential of dissipation, a convex and l.s.c. functional $\tilde{\varphi}(\sigma)$ such that:

$$
\dot{\varepsilon}^{a n}(x, t) \in \partial \tilde{\varphi}[x, t, \sigma(x, t)]
$$

The dependence of $A_{i j k h}$ and $\check{\varphi}$ on the time variable $t$ may model some thermomechanical effects: in many problems, $A$ and $\check{\varphi}$ depend in a known manner on the temperature $T$ which is itself a known function $T(t)$ of the time. Their dependence on the space variables $x$ accounts for a possible nonhomogeneity of the material.

Some examples of potentials: (a) Perfect plasticity. In this case the potential $\tilde{\varphi}$ is the indicator function of a closed convex set $B(x, t)$, called the elasticity set:

$$
\dot{\varepsilon}^{a n}(x, t) \in \partial I_{B(x, t)}(\sigma(x, t))
$$

where $I_{B(x, t)}(\sigma)=0$ if $\sigma \in B(x, t),+\infty$ otherwise (1.4) implies that the stress tensor $\sigma(x, t)$ belongs to $B(x, t)$.

(b) Viscoplasticity I. A particular example of viscoplasticity is the Perzyna law which is the Yosida regularization of the preceding one:

$$
\check{\varphi}_{\mu}(x, t, \tau)=\frac{1}{2 \mu(x, t)}\left\|\tau-\prod_{B(x, t)} \tau\right\|^{2}, \quad \dot{\varepsilon}^{a n}(x, t)=\frac{1}{\mu(x, t)}\left(\sigma(x, t)-\prod_{B(x, t)} \sigma(x, t)\right) .
$$

$\prod_{B(x, t)}$ denotes the projection onto $B(x, t)$ in $R^{6}$ (space of symmetric $3 \times 3$ tensors); $\mu$ is the viscosity. In a formal manner (1.4) is obtained from (1.5) by letting $\mu$ tend to zero. Mercier [17] has used this constitutive law for numerical computations.

(c) Viscoplasticity II. A particular case of viscoelastic material is the Norton-Hoff material introduced by Friâa. The gauge function of $B(x, t)$ is defined by:

$$
j(x, t, \tau)=\operatorname{Inf}\{k \in] 0,+\infty[; \tau \in k B(x, t)\}
$$

Here $j$ is a convex function, positively homogeneous with degree 1 . We set

$$
\tilde{\varphi}_{p}(x, t, \tau)=\frac{1}{p^{\prime}}(j(x, t, \tau))^{p^{\prime}}
$$

where $p^{\prime}=p /(p-1)$. The constitutive law $(1.3)$ is:

$$
\dot{\varepsilon}^{a n}(x, t)=\frac{\partial j}{\partial \sigma}(x, t, \sigma)(j(x, t, \sigma))^{1 /(p-1)} .
$$


The perfectly plastic law (1.4) is formally obtained from (1.6) by letting $p^{\prime}$ tend to $+\infty$. For $p=p^{\prime}=2$ a viscoelastic law of Maxwell is obtained.

2. Conservation laws. In the dynamic case and under the small perturbation assumption the conservation laws lead to:

$$
\rho \ddot{u}_{i}=\sigma_{i j, j}+f_{i} .
$$

In the quasi-static case the inertia forces are neglected:

$$
\sigma_{i j, j}+f_{i}=0 .
$$

The boundary conditions are of mixed type: if $\Omega$ denotes the open set occupied by the body and $\partial \Omega$ its boundary, we assume that the forces are given on a part $\partial \Omega_{F}$ of $\partial \Omega$ and the displacements are imposed on $\partial \Omega_{U}=\partial \Omega-\partial \Omega_{F}$ :

$$
\sigma_{i j} n_{j}=F_{i}^{d}(x, t) \quad \text { on } \quad \partial \Omega_{F}, \quad u_{i}=U_{i}^{d}(x, t) \quad \text { on } \quad \partial \Omega_{U} .
$$

These boundary conditions might be generalized in order to model other problems which impose on the same part of the boundary the normal component of the displacement and the tangential components of the stress (see the problems of extrusion or indentation).

The initial state of the material is regular:

$$
\begin{aligned}
& \sigma(x, 0)=\sigma_{0}(x) \in B(x, 0), \quad \sigma_{0} \cdot n=F^{d} \quad \text { on } \quad \partial \Omega_{F}, \\
& u(x, 0)=u_{0}(x), \quad u_{0}=U^{d} \text { on } \partial \Omega_{U} \text {, } \\
& \left(\sigma_{0}\right)_{i j, j}+f_{i}(0)=0 .
\end{aligned}
$$

For the dynamic problem another piece of data is necessary:

$$
\dot{u}(x, 0)=v_{0}(x) \text {. }
$$

2. The regularized problem: notation and results.

1. Notation. We shall use the spaces $L^{p}(\Omega), H^{s}(\Omega), W^{s, p}(\Omega)$ as defined in [15]. If $Z$ is a vector space of tensors, the subspace of symmetric tensors in $Z$ will be denoted by $Z_{s}$.

$$
H=\left(L^{2}(\Omega)\right)_{s}^{9}=\left\{\tau \mid \tau=\left(\tau_{i j}\right), \quad \tau_{i j}=\tau_{j i}, \quad \tau_{i j} \in L^{2}(\Omega)\right\} .
$$

$H$ is a Hilbert space for the scalar product

$$
(\sigma, \tau)=\int_{\Omega} \sigma_{i j}(x) \tau_{i j}(x) d x, \text { with norm }||
$$

We assume that the dependence of $\tilde{\varphi}$ on the variables $x$ is smooth enough (measurable) to define a convex proper l.s.c. functional by setting

$$
\varphi(t, \tau)=\int_{\Omega} \tilde{\varphi}(x, t, \tau(x)) d x \quad(\forall \tau \in H) .
$$

We denote by $X$ the domain of $\varphi$ in $H$. We assume that $X$ is a vector subspace of $H$, endowed with a different norm $\|\cdot\|_{X}$. Moreover, we assume that $\left(X,\|\cdot\|_{X}\right)$ is a reflexive Banach space containing a space $\left(L^{q}(\Omega)\right)_{s}^{9}$, and $(\mathscr{D}(\bar{\Omega}))_{s}^{9}$ is dense in $X$ for its strong topology. 
Examples. Take for $B(x, t)$ a fixed ball of radius 1 ; then for the Perzyna material

$$
X=H \text { and }\|\|_{X}=\mid \text { | }
$$

and for the Norton-Hoff material

$$
X=\left(L^{p^{\prime}}(\Omega)\right)_{s}^{9} \text { and }\|\cdot\|_{X}=\|\cdot\|_{\left(L^{p^{\prime}}(\Omega)\right) s^{9}} .
$$

The introduction of $X$ is necessary in order to give a sense to the potential $\varphi$. However, this technical point is avoided in most cases since $\varphi$ is often quadratic (see the viscoplasticity) and $X=H$.

We denote by $X^{\prime}$ the dual space of $X$ for the bilinear form

$$
\begin{aligned}
& (\sigma, e)=\int_{\Omega} \sigma_{i j}(x) e_{i j}(x) d x \quad(\forall \sigma \in X)\left(\forall e \in X^{\prime}\right) . \\
& D \tau=\left(\tau_{1 j, j}, \quad \tau_{2 j, j}, \quad \tau_{3 j, j}\right), \\
& \mathscr{H}_{\text {ad }}=\left\{\tau \in X ; \quad D \tau \in\left(L^{3}(\Omega)\right)^{3} ; \quad \tau_{i j} n_{j}=F_{i}^{d} \text { on } \partial \Omega_{F}\right\}^{1} \text {, } \\
& \mathscr{H}_{0}=\left\{\tau \in X ; \quad D \tau \in\left(L^{3}(\Omega)\right)^{3} ; \quad \tau_{i j} n_{j}=0 \quad \text { on } \partial \Omega_{F}\right\}, \\
& V=\left(L^{2}(\Omega)\right)^{3}, \quad W=\left(L^{3 / 2}(\Omega)\right)^{3}, \\
& \mathscr{U}_{\mathrm{ad}}=\left\{u \mid u \in W ; \quad \varepsilon(u) \in X^{\prime} ; \quad u_{i}=U_{i}^{d} \quad \text { on } \quad \partial \Omega_{U}\right\}, \\
& \mathscr{U}_{0}=\left\{u \mid u \in W ; \quad \varepsilon(u) \in X^{\prime} ; \quad u_{i}=0 \text { on } \partial \Omega_{U}\right\}, \\
& \mathscr{V}_{\text {ad }}=\left\{v \mid v \in W ; \quad \varepsilon(v) \in X^{\prime} ; \quad v_{i}=\dot{U}_{i}^{d} \quad \text { on } \quad \partial \Omega_{U}\right\} .
\end{aligned}
$$

Since $X$ contains $\left(L^{q}(\Omega)\right)_{s}^{9}$, the boundary conditions are well defined in the spaces $\mathscr{U}_{\text {ad }}$, $\mathscr{V}_{\text {ad }}$, and $\mathscr{U}_{0}$. Moreover, the density of $(\mathscr{D}(\Omega))_{s}^{9}$ in $X$ implies the Green formula:

$$
(\sigma, \varepsilon(u))+(u, D \sigma)=0 \quad\left(\forall u \in \mathscr{U}_{0}\right)\left(\forall \sigma \in \mathscr{H}_{0}\right) .
$$

2.2 Formulation of the problems.

Problem 1 (dynamic, regular): find $(\sigma, u):[0, T] \rightarrow \mathscr{H}_{\text {ad }} \times \mathscr{U}_{\text {ad }}$ such that:

$$
\begin{gathered}
\frac{d}{d t}[A(t) \sigma]+\partial \varphi(t, \sigma)=\varepsilon(\dot{u}), \\
\rho \ddot{u}-D \sigma=f, \quad \sigma(0)=\sigma_{0}, \quad u(0)=u_{0}, \quad \dot{u}(0)=u_{0} .
\end{gathered}
$$

Problem 2 (quasi-static, regular): find $(\sigma, u):[0, T] \rightarrow \mathscr{H}_{\text {ad }} \times \mathscr{U}_{\text {ad }}$ such that:

$$
\begin{gathered}
\frac{d}{d t}[A(t) \sigma]+\partial \varphi(t, \sigma)=\varepsilon(\dot{u}), \\
-D \sigma=f, \quad \sigma(0)=\sigma_{0}, \quad u(0)=v_{0} .
\end{gathered}
$$

2.3 Hypothesis. We assume that the elastic problems (dynamic and quasi-static) admit unique solutions, $\left(\sigma_{d}^{\mathrm{el}}, u_{d}^{\mathrm{el}}\right),\left(\sigma_{s}^{\mathrm{el}}, u_{s}^{\mathrm{el}}\right)$, twice continuously differentiable with respect to the time, the displacements being in $\left(H^{1}(\Omega)\right)^{3}$.

\footnotetext{
${ }^{1} D \tau \in\left(L^{2}(\Omega)\right)^{2}$ if $\Omega \subset R^{2}$. This will be the case, for simplicity, in Secs. 3.1 and 3.2.
} 
We assume that there exist some constants $\alpha, \beta$ such that:

$$
\begin{array}{ll}
(A(t) \tau, \tau) \geq \alpha|\tau|^{2} \quad(\forall \tau \in H), \\
\left|\frac{\partial^{j} A}{\partial t^{j}}(t) \tau\right| \leq \beta|\tau| \quad(\forall \tau \in H) \quad j=0,1,2 .
\end{array}
$$

The coefficients $A_{i j k h}$ satisfy the symmetry condition

$$
A_{i j k h}=A_{k h i j}=A_{j i k h} \text {. }
$$

The potential $\varphi$ satisfies:

The sets $\{\tau \in X,|\tau| \leq c, \varphi(t, \tau) \leq c\} \quad$ are bounded sets in $X$.

$\partial \varphi$ is a continuous operator from $X$ into $X^{\prime}$ : there exists a real continuous function $L(y)$ such that:

$$
\|\partial \varphi(t, \tau)-\partial \varphi(t, \sigma)\|_{X^{\prime}} \leq L\left(\|\tau-\sigma\|_{X}\right), \quad L(0)=0 .
$$

$\varphi(t, \sigma)$ and $\partial \varphi(t, \sigma)$ are smooth time-dependent functions: $\sigma$ being a fixed element of $X, t \rightarrow \varphi(t, \sigma)$ and $t \rightarrow \partial \varphi(t, \sigma)$ have a Lipshitz regularity with respect to $t$ :

$$
\|\partial \varphi(t, \sigma)-\partial \varphi(s, \sigma)\|_{X^{\prime}}+|\varphi(t, \sigma)-\varphi(s, \sigma)| \leq c_{1}|t-s|\left(\varphi(s, \sigma)+c_{2}\right) .
$$

Remark 2.1. Assumption (2.3) is a minimal assumption; in order to solve the nonelastic problem we must be able to solve the elastic one. Assumptions (2.5) and (2.6) are classical in the theory of linear elasticity. Assumptions (2.7)-(2.9) are formulated on the global potential $\varphi$; they can be formulated as well on the local potential $\tilde{\varphi}$.

2.4 Statement of the results. We shall prove in the following sections:

Proposition 1.: The dynamic problem (2.1) admits a unique solution $(\sigma, u)$ which has the following regularity:

$$
\begin{aligned}
& \sigma \in C^{0}(0, T ; H) \cap L^{\infty}(0, T ; X) ; \quad \dot{\sigma} \in L^{2}(0, T ; H), \\
& u \in C^{1}(0, T ; V) ; \quad v=\dot{u} \in C^{0}(0, T ; V), \\
& \dot{v} \in L^{2}(0, T ; V) .
\end{aligned}
$$

We give to $\rho$ the particular value $\varepsilon$ and we consider the solution $\left(\sigma^{\varepsilon}, u^{\varepsilon}\right)$ of the resulting dynamic problem. Then we prove:

Proposition 2. The quasi-static regular problem admits a unique solution $(\sigma, u)$ with the following regularity:

$$
\begin{aligned}
& \sigma \in C^{0}(0, T ; H) \cap L^{\infty}(0, T ; X) ; \quad \dot{\sigma} \in L^{2}(0, T ; H), \\
& u \in C^{0} ; \quad v=\dot{u} \in L^{\infty}(0, T ; V) .
\end{aligned}
$$

Moreover, if the following assumption (no initial strains) is satisfied:

$$
\varepsilon_{i j}\left(u_{0}\right)=A_{i j k h}(x, 0)\left(\sigma_{0}\right)_{k h}
$$


we let $\rho$ tend to 0 and we state:

$$
\begin{aligned}
& \sigma^{\rho} \rightarrow \sigma \quad \text { in } \quad C^{0}(0, T ; H) \text { strong } \cap L^{\infty}(0, T ; X) \text { weak }^{*}, \\
& \dot{\sigma}^{\rho} \rightarrow \dot{\sigma} \text { in } L^{\infty}(0, T ; H) \text { weak }{ }^{*} \cap \\
& u^{\rho} \rightarrow u \text { in } L^{\infty}(0, T ; v) \text { weak }^{*} \cap L^{2}\left(0, T ; \mathscr{U}_{\text {ad }}\right) \text { weak* } \\
& v^{\rho} \rightarrow v \text { in } L^{\infty}(0, T ; V) \text { weak*. }
\end{aligned}
$$

\section{The dynamic regular problem.}

3.1. We set $\bar{\sigma}=\sigma-\sigma_{d}^{\mathrm{el}}, \bar{u}=u-u_{d}^{\mathrm{el}}, \bar{v}=\dot{u}-\dot{u}_{d}^{\mathrm{el}}, \bar{\varphi}(t, \tau)=\varphi\left(t, \tau+\sigma_{d}^{\mathrm{el}}\right)$. Then the formulation $(2.1)$ is equivalent to: find $(\bar{\sigma}, \bar{v}):[0, T] \rightarrow \mathscr{H}_{0} \times \mathscr{U}_{0}$ such that:

$$
\begin{gathered}
\frac{d}{d t}[A(t) \bar{\sigma}]+\partial \bar{\varphi}(t, \bar{\sigma})=\varepsilon(\bar{v}), \\
\rho \dot{\bar{v}}-D \bar{\sigma}=0, \\
\bar{\sigma}(0)=0, \quad \bar{u}(0)=0, \quad \bar{u}(t)=\int_{0}^{t} \bar{v}(s) d s .
\end{gathered}
$$

For the sake of simplicity we take $\rho=1$ in this section.

Remark 3.1. By the change of variables $U=(\bar{\sigma}, \bar{v})$ and the change of operators:

$$
\mathscr{A}=\left(\begin{array}{l}
A \\
I d
\end{array}\right), \quad \mathscr{B}=\left(\begin{array}{cr}
\partial \bar{\varphi}(t, \cdot) & -\varepsilon \\
-D & 0
\end{array}\right),
$$

the system (3.1) is equivalent to:

$$
\frac{d}{d t}[\mathscr{A}(t) U]+\mathscr{B}(t, U) \ni 0, \quad U(0)=0,
$$

where $(\mathscr{A}(t))$ is a family of linear continuous and selfadjoint operators $(\mathscr{B}(t, \cdot))$ is a family of nonlinear unbounded operators from the Banach space $X \times V$ into its dual, which are monotone on $\mathscr{H}_{0} \times \mathscr{U}_{0}$. It can be proved that $\mathscr{B}(t, \cdot)$ is a maximal operator on its domain $\mathscr{H}_{0} \times \mathscr{U}_{0}$. Then the system (3.3) looks like the known evolution equations of monotone type:

$$
d \tilde{U} / d t+C(t, \tilde{U}) \ni f, \quad U(0)=U_{0} .
$$

However, in order to reduce (3.3) to the form (3.4) we must set

$$
\tilde{U}=A^{1 / 2}(t) U
$$

and the domain of the new operator $C$ depends strongly on $t$.

The results of Attouch and Damlamian [1] do not apply here since the operator $\mathscr{B}$ is not a subdifferential, and the result of Crandall and Pazy [3] gives a solution in a too weak sense. There exist several other results concerning (3.4) but they assume that the domains of the $C(t, \cdot)$ are equal and this is not the case here. Most of these works are concerned with Hilbert frameworks, but we deal with operators defined on a Banach space with values in its dual. Then a direct study is necessary; however, this remark shows that the proofs will be based on the monotonicity of the operators. 
Remark 3.2. We claim that it is possible to assume that

$$
\int_{0}^{T}\left(\frac{d}{d t}[A(t) \tau(t)], \tau(t)\right) d t \geq 0 \quad\left(\forall \tau \in W^{1,2}(0, T ; H)\right) .
$$

If (3.5) does not hold, we multiply the first line of (3.2) by $e^{-2 \lambda t}$ and obtain an equivalent problem for which we examine the integral

$$
\int_{0}^{T} e^{-2 \lambda t}\left(\frac{d}{d t}[A(t) \tau(t)], \tau(t)\right) d t
$$

We set $\tilde{\tau}=e^{-\lambda t} \tau$ and the above quantity becomes:

$$
\int_{0}^{T}\left(\frac{d}{d t}[A(t) \tilde{\tau}(t)], \tilde{\tau}(t)\right) d t+\lambda \int_{0}^{T}(A(t) \tilde{\tau}(t), \tilde{\tau}(t)) d t .
$$

Using the identity

we obtain:

$$
\left(\frac{d}{d t}[A(t) \tilde{\tau}(t)], \tilde{\tau}(t)\right)=\frac{1}{2} \frac{d}{d t}(A(t) \tilde{\tau}(t), \tilde{\tau}(t))+\frac{1}{2}\left(\frac{d A}{d t}(t) \tilde{\tau}(t), \tilde{\tau}(t)\right),
$$

$$
\frac{1}{2}(A(T) \tilde{\tau}(T), \tilde{\tau}(T))+\frac{1}{2} \int_{0}^{T}\left(\frac{d A}{d t}(t) \tilde{\tau}(t), \tilde{\tau}(t)\right) d t+\lambda \int_{0}^{T}(A(t) \tilde{\tau}(t), \tilde{\tau}(t)) d t .
$$

This term is positive for $\lambda$ large enough by assumptions (2.4) and (2.5).

3.2. Implicit scheme for (3.2). We use a finite difference discretization in time. Let $k=T / N$ where $N$ is a positive integer, $t^{n}=n k, n=0,1, \ldots, N, \sigma^{n}=\sigma\left(t_{n}\right), \partial \bar{\varphi}_{n}(\tau)=$ $\partial \bar{\varphi}\left(t_{n}, \tau\right), A_{n}=A\left(t_{n}\right)$. We now pose the implicit problem:

$$
\bar{\sigma}(0)=0, \quad \bar{v}(0)=0 .
$$

$\bar{\sigma}^{n+1}, \bar{v}^{n+1}$ are defined by induction as solution of:

$$
\begin{gathered}
\bar{\sigma}^{n+1} \in \mathscr{H}_{0}, \quad \bar{v}^{n+1} \in \mathcal{U}_{0}, \\
A_{n+1} \bar{\sigma}^{n+1}-A_{n} \bar{\sigma}^{n}+k \partial \bar{\varphi}_{n+1}\left(\bar{\sigma}^{n+1}\right)=k \varepsilon\left(\bar{v}^{n+1}\right), \\
\bar{v}^{n+1}-\bar{v}^{n}-k D \bar{\sigma}^{n+1}=0 .
\end{gathered}
$$

A solution of (3.6), if it exists, is a saddle-point for the Lagrangian

$$
\mathscr{L}(\bar{\tau}, \bar{w})=\frac{1}{2}\left(A_{n+1} \bar{\tau}, \bar{\tau}\right)-\left(A_{n} \bar{\sigma}^{n}, \bar{\tau}\right)+k \bar{\varphi}_{n+1}(\bar{\tau})-k(\varepsilon(\bar{w}), \bar{\tau})-\frac{1}{2}|\bar{w}|^{2}+\left(\bar{v}^{n}, \bar{w}\right)
$$

on $\mathscr{H}_{0} \times \mathscr{U}_{0}$. This Lagrangian does not have a sufficiently strong growth at infinity on $\mathscr{H}_{0} \times \mathscr{U}_{0}$, and it is not possible to prove directly the existence of a saddle point for $\mathscr{L}$. But we can consider an augmented Lagrangian: if $\mathbf{J}$ is a duality application between $X^{\prime}$ and $X$ satisfying

we set:

$$
\|\mathbf{J}(e)\|_{X}=\|e\|_{X^{\prime}}, \quad \mathbf{J}(\lambda e)=\lambda \mathbf{J}(e), \quad(\mathbf{J}(e), e)=\|\mathbf{J}(e)\|_{X}\|e\|_{X^{\prime}}
$$

$$
\begin{aligned}
\Psi(e) & =(\mathbf{J}(e), e) \\
\mathscr{L}_{\eta}(\bar{\tau}, \bar{w}) & =\mathscr{L}(\bar{\tau}, \bar{w})+\frac{\eta}{2}|D \bar{\tau}|^{2}-\eta \Psi(\varepsilon(\bar{w})) .
\end{aligned}
$$


It follows from an abstract theorem [6, p. 161] that $\mathscr{L}_{\eta}$ admits a saddle-point $\left(\bar{\sigma}_{\eta}^{n+1}, \bar{v}_{\eta}^{n+1}\right)$ on $\mathscr{H}_{0} \times \mathscr{U}_{0}$, which moreover satisfies

$$
\begin{aligned}
\left(A_{n+1} \bar{\sigma}_{\eta}^{n+1}-A_{n} \bar{\sigma}^{n}+k \partial \bar{\varphi}_{n+1}\left(\bar{\sigma}_{\eta}^{n+1}\right), \bar{\tau}\right)+\eta\left(D \bar{\sigma}_{\eta}^{n+1}, D \bar{\tau}\right)=k\left(\varepsilon\left(\bar{v}_{\eta}^{n+1}\right), \bar{\tau}\right), & \\
& \left(\bar{v}_{\eta}^{n+1}-\bar{v}_{\eta}^{n}-k D \bar{\sigma}_{\eta}^{n+1}, \bar{w}\right)+\eta\left(\partial \Psi\left(\varepsilon\left(\bar{v}_{\eta}^{n+1}\right)\right), \varepsilon(\bar{w})\right)=0 .
\end{aligned}
$$

Taking now $\tau=\bar{\sigma}_{\eta}^{n+1}$ and $\bar{w}=\bar{v}_{\eta}^{n+1}$ as test functions, we get:

$$
\begin{aligned}
\left(A_{n+1} \bar{\sigma}_{\eta}^{n+1}, \bar{\sigma}_{\eta}^{n+1}\right)+\eta \mid D \bar{\sigma}_{\eta}^{n+1} & \left.\right|^{2}+k\left(\partial \bar{\varphi}_{n+1}\left(\bar{\sigma}_{\eta}^{n+1}\right), \bar{\sigma}_{\eta}^{n+1}\right)+\left|\bar{v}_{\eta}^{n+1}\right|^{2} \\
& +\left(\partial \Psi\left(\varepsilon\left(\bar{v}_{\eta}^{n+1}\right)\right), \varepsilon\left(\bar{v}_{\eta}^{n+1}\right)\right)=\left(\bar{v}_{\eta}^{n}, \bar{v}_{\eta}^{n+1}\right)+\left(A_{n} \bar{\sigma}^{n}, \bar{\sigma}_{\eta}^{n+1}\right) .
\end{aligned}
$$

Using the inequalities

$$
\begin{aligned}
(\partial \Psi(e), e) & \geq \Psi(e)=\|e\|_{X^{\prime}}^{2} \\
\left(\partial \bar{\varphi}_{n+1}\left(\bar{\sigma}_{\eta}^{n+1}\right), \bar{\sigma}_{\eta}^{n+1}\right) & =\left(\partial \varphi_{n+1}\left(\sigma_{\eta}^{n+1}\right), \sigma_{\eta}^{n+1}-\left(\sigma_{d}^{\mathrm{el}}\right)^{n+1}\right) \\
& \geq \varphi_{n+1}\left(\sigma_{\eta}^{n+1}\right)-\varphi_{n+1}\left(\left(\sigma_{d}^{\mathrm{el}}\right)^{n+1}\right) \geq \Rightarrow \varphi_{n+1}\left(\left(\sigma_{d}^{\mathrm{el}}\right)^{n+1}\right),
\end{aligned}
$$

we get:

$\bar{\sigma}_{\eta}^{n+1}$ is a bounded sequence in $H$ independently of $\eta$,

$\bar{v}_{\eta}^{n+1}$ is a bounded sequence in $V$ independently of $\eta$.

Then we deduce from (3.7):

$$
\bar{\varphi}_{n+1}\left(\bar{\sigma}_{\eta}^{n+1}\right) \leq\left(\partial \bar{\varphi}_{n+1}\left(\bar{\sigma}_{\eta}^{n+1}\right), \bar{\sigma}_{\eta}^{n+1}\right)+\bar{\varphi}_{n+1}(0) \leq c,
$$

and from assumption (2.7):

$$
\bar{\sigma}_{\eta}^{n+1} \text { is a bounded sequence in } X \text { independently of } \eta \text {. }
$$

The continuity of $\partial \bar{\varphi}_{n+1}$ from $X$ into $X^{\prime}$ provides:

$$
\partial \bar{\varphi}_{n+1}\left(\bar{\sigma}_{\eta}^{n+1}\right) \text { is a bounded sequence in } X^{\prime} \text { independently of } \eta \text {. }
$$

We also derive from (3.7):

$$
\eta^{1 / 2} D \bar{\sigma}_{\eta}^{n+1} \text { is a bounded sequence in } V \text { independently of } \eta \text {. }
$$

Then the inequality.

$$
\eta\left\|\varepsilon\left(\bar{v}_{\eta}^{n+1}\right)\right\|^{2}=\eta \Psi\left(\varepsilon\left(\bar{v}_{\eta}^{n+1}\right)\right) \leq \eta\left(\partial \Psi\left(\varepsilon\left(\bar{v}_{\eta}^{n+1}\right)\right), \varepsilon\left(\bar{v}_{\eta}^{n+1}\right)\right)
$$

leads to

$$
\eta^{1 / 2} \varepsilon\left(\bar{v}_{\eta}^{n+1}\right) \text { is a bounded sequence in } X^{\prime} \text { independently of } \eta \text {. }
$$

Then there exists $\bar{\sigma}^{n+1} \in X, \vec{v}^{n+1} \in V, \chi \in X^{\prime}$ such that

$$
\begin{array}{rlrl}
\bar{\sigma}_{\eta}^{n+1} & \rightarrow \bar{\sigma}^{n+1} & \text { in } X \text { weak*, } \\
\bar{v}_{\eta}^{n+1} & \rightarrow \bar{v}^{n+1} & \text { in } \quad V \text { weak, } \\
\partial \bar{\varphi}_{n+1}\left(\bar{\sigma}_{\eta}^{n+1}\right) & \rightarrow \chi & & \text { in } \quad X^{\prime} \text { weak*. }
\end{array}
$$

In order to take the limit of the above equations, we note that

$$
\eta \partial \Psi\left(\varepsilon\left(\bar{v}_{\eta}^{n+1}\right)\right)=\eta^{1 / 2}\left(\eta^{1 / 2}\left\|\varepsilon\left(\bar{v}_{\eta}^{n+1}\right)\right\|_{X^{\prime}}\right) \partial \Psi\left(e_{\eta}^{n+1}\right)
$$


where $e_{\eta}^{n+1}=\varepsilon\left(\bar{v}_{\eta}^{n+1}\right) /\left\|\varepsilon\left(\bar{v}_{\eta}^{n+1}\right)\right\|$. But

$$
\left(\partial \Psi\left(e_{\eta}^{n+1}\right), e\right) \leq \Psi\left(e_{\eta}^{n+1}+e\right)-\Psi\left(e_{\eta}^{n+1}\right)=\left\|e_{\eta}^{n+1}+e\right\|_{X^{\prime}}^{2}-\left\|e_{\eta}^{n+1}\right\|_{X^{\prime}}^{2} .
$$

Hence $\left\|\partial \Psi\left(e_{\eta}^{n+1}\right)\right\|_{X} \leq 3$ and using (3.12) we have

$$
\lim _{\eta \rightarrow 0} \eta \partial \Psi\left(\varepsilon\left(\bar{v}_{\eta}^{n+1}\right)\right)=0 \text { in } X .
$$

$\bar{\sigma}^{n+1}, \bar{v}^{n+1}, \chi$ are solutions in the sense of $\mathscr{D}^{\prime}(\Omega)$ of

$$
A_{n+1} \bar{\sigma}^{n+1}-A_{n} \bar{\sigma}^{n}+k \chi=k \varepsilon\left(\bar{v}^{n+1}\right), \quad \bar{v}^{n+1}-\bar{v}^{n}-k D \bar{\sigma}^{n+1}=0 .
$$

These equations show that $\varepsilon\left(\bar{v}^{n+1}\right) \in X^{\prime}$, and $D \bar{\sigma}^{n+1} \in V$. In order to prove that $\bar{v}^{n+1} \in \mathscr{U}_{0}$ and $\bar{\sigma}^{n+1} \in \mathscr{H}_{0}$, it is sufficient to check that the boundary conditions are satisfied. For every $\tau \in(\mathscr{D}(\bar{\Omega}))_{s}^{9} \cap \mathscr{H}_{0}$ we have

$$
\begin{gathered}
\left(\varepsilon\left(\bar{v}_{\eta}^{n+1}\right), \tau\right)+\left(\bar{v}_{\eta}^{n+1}, D \tau\right)=0, \\
k\left(\bar{v}_{\eta}^{n+1}, D \tau\right)+\left(A_{n+1} \bar{\sigma}_{\eta}^{n+1}-A_{n} \bar{\sigma}^{n}, \tau\right)+\left(k \partial \bar{\varphi}_{n+1}\left(\bar{\sigma}_{\eta}^{n+1}\right), \tau\right)+\eta\left(D \bar{\sigma}_{\eta}^{n+1}, D \tau\right)=0 .
\end{gathered}
$$

Using (3.13)-(3.17) we conclude that

$$
\left(\bar{v}^{n+1}, D \tau\right)+\left(\varepsilon\left(\bar{v}^{n+1}\right), \tau\right)=0
$$

which shows that $\bar{v}^{n+1} \in \mathscr{U}_{0}$. Similar reasoning shows that $\bar{\sigma}^{n+1} \in \mathscr{H}_{0}$. It remains to prove that $\chi=\partial \bar{\varphi}_{n+1}\left(\bar{\sigma}^{n+1}\right)$, or equivalently:

$$
\left(\chi-\partial \bar{\varphi}_{n+1}(\tau), \bar{\sigma}^{n+1}-\tau\right) \geq 0 \quad(\forall \tau \in X) .
$$

We know that $k\left(\partial \bar{\varphi}_{n+1}\left(\bar{\sigma}_{\eta}^{n+1}\right)-\partial \bar{\varphi}_{n+1}(\tau), \bar{\sigma}_{\eta}^{n+1}-\tau\right) \geq 0$. Then

$$
\begin{array}{r}
\left(A_{n} \bar{\sigma}^{n}, \bar{\sigma}_{\eta}^{n+1}\right)-\left(A_{n+1} \bar{\sigma}_{\eta}^{n+1}, \bar{\sigma}_{\eta}^{n+1}\right)+\left(\bar{v}^{n}, \bar{v}_{\eta}^{n+1}\right)-\left|\bar{v}_{\eta}^{n+1}\right|^{2}-\eta\left(\partial \Psi\left(\varepsilon\left(\bar{v}_{\eta}^{n+1}\right)\right), \varepsilon\left(\bar{v}_{\eta}^{n+1}\right)\right) \\
-\eta\left|D \bar{\sigma}_{\eta}^{n+1}\right|^{2} \geq k\left(\partial \bar{\varphi}_{n+1}(\tau), \bar{\sigma}_{\eta}^{n+1}-\tau\right)+k\left(\partial \bar{\varphi}_{n+1}\left(\bar{\sigma}_{\eta}^{n+1}\right), \tau\right) .
\end{array}
$$

By the lower semi-continuity of the functional $\tau \rightarrow\left(A_{n+1} \tau, \tau\right)$ we get:

$$
\begin{aligned}
&\left(A_{n} \bar{\sigma}^{n}, \bar{\sigma}^{n+1}\right)-\left(A_{n+1} \bar{\sigma}^{n+1}, \bar{\sigma}^{n+1}\right)+\left(\bar{v}^{n}, \bar{v}^{n+1}\right)-\left|\bar{v}^{n+1}\right|^{2} \\
& \geq k\left(\partial \bar{\varphi}_{n+1}(\tau), \bar{\sigma}^{n+1}-\tau\right)+k(\chi, \tau) .
\end{aligned}
$$

The first term of this inequality is $k\left(\chi, \bar{\sigma}^{n+1}\right)$ and we get (3.18). Then we have proved the existence of a solution for the implicit scheme (3.6).

3.2 The continuous problem. We define an element $\bar{\sigma}_{N}$ of $L^{\infty}(0, T ; X)$, and an element $\bar{v}_{N}$ of $L^{\infty}(0, T ; V)$ by setting:

$$
\begin{array}{ll}
\bar{\sigma}_{N}(t)=\bar{\sigma}^{n}+\frac{\left(t-t_{n}\right)\left(\bar{\sigma}^{n+1}-\bar{\sigma}^{n}\right)}{\left(t_{n+1}-t_{n}\right)} \quad \text { if } t \in\left[t_{n}, t_{n+1}\right], \\
\bar{v}_{N}(t)=\bar{v}^{n}+\frac{\left(t-t_{n}\right)\left(\bar{v}^{n+1}-\bar{v}^{n}\right)}{\left(t_{n+1}-t_{n}\right)} \quad \text { if } t \in\left[t_{n}, t_{n+1}\right] .
\end{array}
$$

Note that $\bar{\sigma}_{N}\left(t_{n}\right)=\bar{\sigma}^{n} ; \bar{v}_{N}\left(t_{n}\right)=\bar{v}^{n}$. We define a family of convex functionals $\bar{\varphi}_{N}$ by:

$$
\bar{\varphi}_{N}(t, \tau)=\bar{\varphi}_{n}(\tau) \quad \text { if } \quad t \in\left[t_{n}, t_{n+1}\right]
$$


Note that by assumption (2.9):

$$
\lim _{N \rightarrow+\infty} \partial \bar{\varphi}_{N}(t, \tau)=\partial \bar{\varphi}(t, \tau) \text { in } L^{\infty}\left(0, T ; X^{\prime}\right) .
$$

A priori estimates I. Take $\bar{\sigma}^{n+1}$ and $\bar{v}^{n+1}$ as test functions:

$$
\left(A_{n+1} \bar{\sigma}^{n+1}-A_{n} \bar{\sigma}^{n}, \bar{\sigma}^{n+1}\right)+k\left(\partial \bar{\varphi}_{n+1}\left(\bar{\sigma}^{n+1}\right), \bar{\sigma}^{n+1}\right)+\left(\bar{v}^{n+1}-\bar{v}^{n}, \bar{v}^{n+1}\right)=0 .
$$

Use the following equalities:

$$
\begin{aligned}
& \left(A_{n+1} \bar{\sigma}^{n+1}-A_{n} \bar{\sigma}^{n}, \bar{\sigma}^{n+1}\right) \\
& =\frac{1}{2}\left(A_{n+1} \bar{\sigma}^{n+1}, \bar{\sigma}^{n+1}\right)-\frac{1}{2}\left(A_{n} \bar{\sigma}^{n}, \bar{\sigma}^{n}\right)+\frac{1}{2}\left(A_{n}\left(\bar{\sigma}^{n+1}-\bar{\sigma}^{n}\right), \bar{\sigma}^{n+1}-\bar{\sigma}^{n}\right) \\
& -\frac{1}{2}\left(\left(A_{n+1}-A_{n}\right) \bar{\sigma}^{n+1}, \bar{\sigma}^{n+1}\right), \\
& \left(\partial \bar{\varphi}_{n+1}\left(\bar{\sigma}^{n+1}\right), \bar{\sigma}^{n+1}\right) \geq \partial \varphi_{n+1}\left(\sigma^{n+1}\right)-\partial \varphi_{n+1}\left(\left(\sigma_{d}^{\text {el }}\right)^{n+1}\right) \geq-\partial \varphi_{n+1}\left(\left(\sigma_{d}^{\text {el }}\right)^{n+1}\right) \geq-c, \\
& \left(\bar{v}^{n+1}-\bar{v}^{n}, \bar{v}^{n+1}\right)=\frac{1}{2}\left|\bar{v}^{n+1}\right|^{2}-\frac{1}{2}\left|\bar{v}^{n}\right|^{2}+\frac{1}{2}\left|\bar{v}^{n+1}-\bar{v}^{n}\right|^{2} .
\end{aligned}
$$

After summation of the successive equations (3.19) we get:

$$
\frac{1}{2}\left(A_{m} \bar{\sigma}^{m}, \bar{\sigma}^{m}\right)+\frac{1}{2}\left|\bar{v}^{m}\right|^{2} \leq c+\frac{1}{2} \sum_{n=1}^{m-1} k\left\|\frac{A_{n+1}-A_{n}}{k}\right\|_{L^{\infty}}\left|\bar{\sigma}^{n}\right|^{2}+\frac{1}{2}\left\|A_{m+1}-A_{m}\right\|_{L^{\infty}}\left|\bar{\sigma}^{m+1}\right|^{2} .
$$

For $k$ small enough, we get from assumption (2.5) and a discrete version of the Gronwall lemma:

$$
\begin{aligned}
& \bar{\sigma}_{N} \text { is a bounded sequence in } L^{\infty}(0, T ; H), \\
& \bar{v}_{N} \text { is a bounded sequence in } L^{\infty}(0, T ; V) .
\end{aligned}
$$

A priori estimates II. We take the difference between the two systems (3.6) written at times $t_{n}$ and $t_{n+1}$ :

$$
\begin{aligned}
A_{n+2} \bar{\sigma}^{n+2} & -2 A_{n+1} \bar{\sigma}^{n+1}+A_{n} \bar{\sigma}^{n}+k\left(\partial \bar{\varphi}_{n+2}\left(\bar{\sigma}^{n+2}\right)-\partial \bar{\varphi}_{n+1}\left(\bar{\sigma}^{n+1}\right)\right) \\
= & k\left(\varepsilon\left(\bar{v}^{n+2}\right)-\varepsilon\left(\bar{v}^{n+1}\right)\right), \quad \bar{v}^{n+2}-2 \bar{v}^{n+1}+\bar{v}^{n}-k\left(D \bar{\sigma}^{n+2}-D \bar{\sigma}^{n+1}\right)=0 .
\end{aligned}
$$

Take $\bar{\sigma}^{n+2}-\bar{\sigma}^{n+1}$ and $\bar{v}^{n+2}-\bar{v}^{n+1}$ as test functions. An easy but rather tedious computation shows that:

$$
\begin{aligned}
&\left(A_{n+2} \bar{\sigma}^{n+2}-\right.\left.2 A_{n+1} \bar{\sigma}^{n+1}+A_{n} \bar{\sigma}^{n}, \bar{\sigma}^{n+2}-\bar{\sigma}^{n+1}\right)=\frac{1}{2}\left(A_{n+2}\left(\bar{\sigma}^{n+2}-\bar{\sigma}^{n+1}\right), \bar{\sigma}^{n+2}-\bar{\sigma}^{n+1}\right) \\
&-\frac{1}{2}\left(A_{n+1}\left(\bar{\sigma}^{n+1}-\bar{\sigma}^{n}\right), \bar{\sigma}^{n+1}-\bar{\sigma}^{n}\right) \\
&+\frac{1}{2}\left(\left(A_{n+1}-A_{n}\right)\left(\bar{\sigma}^{n+1}-\bar{\sigma}^{n}\right), \bar{\sigma}^{n+2}-\bar{\sigma}^{n+1}\right) \\
&+\frac{1}{2}\left(\left(A_{n+2}-2 A_{n+1}+A_{n}\right) \bar{\sigma}^{n+1}, \bar{\sigma}^{n+2}-\bar{\sigma}^{n+1}\right) \\
&+\frac{1}{2}\left(A_{n+2} \bar{\sigma}^{n+2}-2 A_{n+1} \bar{\sigma}^{n+1}+A_{n} \bar{\sigma}^{n}, \bar{\sigma}^{n+2}-2 \bar{\sigma}^{n+1}+\bar{\sigma}^{n}\right) \\
&+\frac{1}{2}\left(\left(A_{n+2}-A_{n+1}\right)\left(\bar{\sigma}^{n+2}-\bar{\sigma}^{n+1}\right), \bar{\sigma}^{n+1}-\bar{\sigma}^{n}\right) \\
&+\frac{1}{2}\left(\left(A_{n+1}-A_{n}\right)\left(\bar{\sigma}^{n+1}-\bar{\sigma}^{n}\right), \bar{\sigma}^{n+1}-\bar{\sigma}^{n}\right) \\
&+\frac{1}{2}\left(\left(A_{n+2}-2 A_{n+1}+A_{n}\right) \bar{\sigma}^{n+1}, \bar{\sigma}^{n+1}-\bar{\sigma}^{n}\right), \\
&\left(\bar{v}^{n+2}-2 \bar{v}^{n+1}+\bar{v}^{n}, \bar{v}^{n+2}-\bar{v}^{n+1}\right)=\frac{1}{2}\left|\bar{v}^{n+2}-\bar{v}^{n+1}\right|^{2} \\
& \quad-\frac{1}{2}\left|\bar{v}^{n+1}-\bar{v}^{n}\right|^{2}+\frac{1}{2}\left|\bar{v}^{n+2}-2 \bar{v}^{n+1}+\bar{v}^{n}\right|^{2} .
\end{aligned}
$$


After division of (3.22) by $k^{2}$, summation over $n$ and a little work:

$$
\left(A_{m+1}\left(\frac{\bar{\sigma}^{m+1}-\bar{\sigma}^{m}}{k}\right), \frac{\bar{\sigma}^{m+1}-\bar{\sigma}^{m}}{k}\right)+\left|\frac{\bar{v}^{m+1}-\bar{v}^{m}}{k}\right|^{2} \leq c+\sum_{n=0}^{m-1} k\left|\frac{\bar{\sigma}^{n+1}-\bar{\sigma}^{n}}{k}\right|^{2} .
$$

The discrete version of the Gronwall lemma gives:

$$
\begin{gathered}
\dot{\bar{\sigma}}_{N} \text { is a bounded sequence in } L^{\infty}(0, T ; H), \\
\dot{\bar{v}}_{N} \text { is a bounded sequence in } L^{\infty}(0, T ; V) .
\end{gathered}
$$

Using (3.19), (3.23) and (3.24) we see that:

$$
\left(\partial \bar{\varphi}_{n+1}\left(\bar{\sigma}^{n+1}\right), \bar{\sigma}^{n+1}\right) \text { is a bounded sequence in } L^{\infty}(0, T) .
$$

Then, $\varphi_{n+1}\left(\sigma^{n+1}\right) \leq\left(\partial \bar{\varphi}_{n+1}\left(\bar{\sigma}^{n+1}\right), \bar{\sigma}^{n+1}\right)+\varphi_{n+1}\left(\left(\sigma_{d}^{\text {el }}\right)^{n+1}\right) \leq c$, and we conclude from (3.20) and assumption (2.7):

$$
\bar{\sigma}_{N} \text { is a bounded sequence in } L^{\infty}(0, T ; X),
$$

Then by the continuity of $\partial \bar{\varphi}$ :

$$
\partial \bar{\varphi}_{N}\left(\bar{\sigma}_{N}\right) \text { is a bounded sequence in } L^{\infty}\left(0, T ; X^{\prime}\right)
$$

Then there exists $\bar{\sigma} \in L^{\infty}(0, T ; X) \cap W^{1, \infty}(0, T ; H), \quad \bar{v} \in W^{1, \infty}(0, T ; V)$ and $\chi \in L^{\infty}\left(0, T ; X^{\prime}\right)$ such that:

$$
\begin{aligned}
& \bar{\sigma}_{N} \rightarrow \bar{\sigma} \quad \text { in } \quad L^{\infty}(0, T ; X) \text { weak }^{*} \cap L^{\infty}(0, T ; H) \text { weak* }{ }^{*}, \\
& \dot{\bar{\sigma}}_{N} \rightarrow \dot{\bar{\sigma}} \quad \text { in } L^{\infty}(0, T ; H) \text { weak* }^{*} \text {, } \\
& \bar{v}_{N} \rightarrow \bar{v} \quad \text { in } L^{\infty}(0, T ; V) \text { weak*, } \\
& \dot{\bar{v}}_{N}-\dot{\bar{v}} \quad \text { in } L^{\infty}(0, T ; V) \text { weak* }^{*} \text {, } \\
& \partial \bar{\varphi}_{N}\left(\bar{\sigma}_{N}\right)-\chi \quad \text { in } L^{\infty}\left(0, T ; X^{\prime}\right) \text { weak* }
\end{aligned}
$$

$\bar{\sigma}, \bar{v}$ and $\chi$ are solutions of:

$$
\frac{d}{d t}(A \bar{\sigma})+\chi=\varepsilon(\bar{v}), \quad d \bar{v} / d t-D \bar{\sigma}=0 .
$$

$D \bar{\sigma}_{N}$ and $\varepsilon\left(\bar{v}_{N}\right)$ are respectively bounded in $L^{\infty}(0, T ; V)$ and $L^{\infty}\left(0, T ; X^{\prime}\right)$; then $\bar{\sigma}_{N}$ and $\bar{v}_{N}$ are bounded in $L^{\infty}\left(0, T ; \mathscr{H}_{0}\right)$ and $L^{\infty}\left(0, T ; \mathscr{U}_{0}\right)$. It can also be shown by use of Green's formula (on the variable $t$ ) that $\bar{\sigma}(0)=0, \bar{v}(0)=0$. It remains to show that $\chi=\partial \bar{\varphi}(\bar{\sigma})$, or:

$$
\left(\forall \tau \in L^{\infty}(0, T ; X)\right) \int_{0}^{T}(\chi-\partial \bar{\varphi}(\tau), \bar{\sigma}-\tau) d t \geq 0 .
$$

We know that $\sum_{n=0}^{N-1} k\left(\partial \bar{\varphi}_{n+1}\left(\bar{\sigma}^{n+1}\right)-\partial \bar{\varphi}_{n+1}(\tau), \bar{\sigma}^{n+1}-\tau\right) \geq 0$, i.e.:

$$
\sum_{n=0}^{N-1} k\left[\left(\partial \bar{\varphi}_{n+1}\left(\bar{\sigma}^{n+1}\right), \bar{\sigma}^{n+1}\right)-\left(\partial \bar{\varphi}_{n+1}(\tau), \bar{\sigma}^{n+1}-\tau\right)-\left(\partial \bar{\varphi}_{n+1}\left(\bar{\sigma}^{n+1}\right), \tau\right)\right] \geq 0 .
$$

The limit of the last two terms in (3.29) is easily evaluated:

$$
-\int_{0}^{T}[(\partial \bar{\varphi}(\tau), \bar{\sigma}-\tau)+(\chi, \tau)] d t
$$


The first term is equal to

$$
\begin{aligned}
& -\sum_{n=0}^{N-1}\left[\left(A_{n+1} \bar{\sigma}^{n+1}-\bar{\sigma}^{n}, \bar{\sigma}^{n+1}\right)+\left(\bar{v}^{n+1}-\bar{v}^{n}, \bar{v}^{n+1}\right)\right] \\
& \quad \leq-\frac{1}{2}\left(A_{N} \bar{\sigma}^{N}, \bar{\sigma}^{N}\right)-\frac{1}{2}\left|\bar{v}^{N}\right|^{2}+\frac{1}{2} \sum_{n=0}^{N-1} k\left(\left(\frac{A_{n+1}-A_{n}}{k}\right) \bar{\sigma}_{N}^{n+1}, \bar{\sigma}_{N}^{n+1}\right) .
\end{aligned}
$$

We note that the discrete version of inequality (3.5) is that the operator

$$
\tau \rightarrow \frac{1}{2}\left(A_{N} \tau, \tau\right)-\frac{1}{2} \sum_{n=0}^{N-1} k\left(\left(\frac{A_{n+1}-A_{n}}{k}\right) \tau^{n+1}, \tau^{n+1}\right)
$$

is a monotone operator. Then by monotonicity:

$$
\begin{aligned}
& \underset{N}{\lim \inf } \frac{1}{2}\left(A_{N} \bar{\sigma}^{N}, \bar{\sigma}^{N}\right)+\frac{1}{2}\left|\bar{v}^{N}\right|^{2}-\frac{1}{2} \sum_{n=0}^{N-1} k\left(\left(\frac{A_{n+1}-A_{n}}{k}\right) \bar{\sigma}_{N}^{n+1}, \bar{\sigma}_{N}^{n+1}\right) \\
& \quad \geq \frac{1}{2}(A \bar{\sigma}(T), \bar{\sigma}(T))+\frac{1}{2}|\bar{v}(T)|^{2}-\frac{1}{2} \int_{0}^{T}\left(\frac{d A}{d t} \bar{\sigma}(t), \bar{\sigma}(t)\right) d t \\
& \quad \geq \int_{0}^{T}\left[\left(\frac{d \bar{v}}{d t}, \bar{v}\right)+\left(\frac{d(A \bar{\sigma})}{d t}, \bar{\sigma}\right)\right] d t \\
& \geq-\int_{0}^{T}(\chi, \bar{\sigma}) d t .
\end{aligned}
$$

Then:

$$
\int_{0}^{T}(\chi, \bar{\sigma}) \geq \lim \sup _{N} \sum_{n=0}^{N-1} k\left(\partial \bar{\varphi}_{n+1}\left(\bar{\sigma}^{n+1}\right), \bar{\sigma}^{n+1}\right) \geq \int_{0}^{T}[(\chi, \tau)+(\partial \bar{\varphi}(\tau), \bar{\sigma}-\tau)] d t
$$

which proves (3.28), and ends the proof of Proposition 1.

4. The quasi-static regular problem. In the general case the dynamic and the quasistatic behaviors of a dissipative material are quite different. In dynamic loading waves are propagated with a velocity which depends on $1 / \rho$; this velocity tends to $+\infty$ when $\rho$ tends to 0 , and this shows that the quasi-static solutions cannot be obtained from the dynamic ones simply by neglecting the inertia terms. Nevertheless, this result is valid if there is no initial stress, since in this case the amplitude of propagating waves tends to 0 with $\rho$. We shall show later that under the assumption (2.10) the dynamic problem is a regularization of the quasi-static one.

Assumption (2.10) is not a realistic one and we must prove Proposition 2 independently on the results obtained in the dynamic case. The proof by discretization in time is a straightforward adaptation of the reasonings in Sec. 3.

We set:

$$
\begin{aligned}
S(t) & =\left\{\tau \in \mathscr{H}_{\text {ad }}(t) ; D \tau+f=0 \text { in } \Omega\right\}, \\
S_{0} & =\left\{\tau \in \mathscr{H}_{0} ; \quad D \tau=0 \text { in } \Omega\right\}
\end{aligned}
$$

and we consider the following problem: $\left(\sigma^{n}, u^{n}\right)$ being defined by induction, find $\left(\sigma^{n+1}, u^{n+1}\right)$ such that

$$
\sigma^{n+1} \in S\left(t^{n+1}\right) ; \quad A_{n+1} \sigma^{n+1}-A_{n} \sigma^{n}+\delta t \partial \varphi\left(\sigma^{n+1}\right)=\varepsilon\left(u^{n+1}\right)-\varepsilon\left(u^{n}\right) .
$$


The solution $\sigma^{n+1}$ of this problem is the minimizer on $S\left(t^{n+1}\right)$ of the convex functional

$$
\frac{1}{2}\left(A_{n+1} \tau, \tau\right)-\left(A_{n \sigma m}, \tau\right)+\delta t \varphi(\tau)-\int_{\partial \Omega_{U}} \tau \cdot n\left(\bar{v}^{d, n+1}-\bar{v}^{d, n}\right) d y .
$$

Then we set $e^{n+1}=A_{n+1} \sigma^{n+1}-A_{n} \sigma^{n}+\delta t \partial \varphi\left(\sigma^{n+1}\right)+\varepsilon\left(u^{n}\right)$. $e^{n+1}$ is orthogonal to $S_{0} \cap(\mathscr{D}(\Omega))_{s}^{9}$ and we deduce from a theorem of [24] that $e^{n+1}$ is the deformation field of a displacement $u^{n+1}$. From the minimizing property of $\sigma^{n+1}$ we deduce that $u^{n+1}$ satisfies the boundary conditions on $\partial \Omega_{U}$. Then we let the time discretization step tend to 0 ; by means of the same reasoning as in Sec. 3.2 we prove Proposition 2.

Let us now prove in details the second part of this proposition. Assumption (2.10) was missing in [5] and is the condition of complete correctness.

We consider the sequence $\left(\sigma^{\rho}, v^{\rho}\right)$ of solutions of $(2.1)$, and the solution $\left(\sigma_{s}^{\text {el }}, v_{s}^{\text {el }}\right)$ of the elastic static problem:

$$
\begin{gathered}
\frac{d}{d t}\left(A \sigma_{s}^{\mathrm{el}}\right)=\varepsilon\left(v_{s}^{\mathrm{el}}\right), \quad-D \sigma_{s}^{\mathrm{el}}=f(t), \\
\sigma_{s}^{\mathrm{el}}(t) \in \mathscr{H}_{\mathrm{ad}}(t), \quad v_{s}^{\mathrm{el}} \in \mathscr{V}_{\mathrm{ad}}(t), \quad \sigma_{s}^{\mathrm{el}}(0)=\sigma_{0} .
\end{gathered}
$$

Let us set $\tilde{\sigma}^{\rho}=\sigma^{\rho}-\sigma_{s}^{\mathrm{el}}, \tilde{v}^{\rho}=v^{\rho}-v_{s}^{\mathrm{el}}, \tilde{\varphi}\left(t, \tilde{\sigma}^{\rho}\right)=\varphi\left(t, \sigma^{\rho}\right)$. Then

$$
\begin{gathered}
\tilde{\sigma}^{\rho} \in \mathscr{H}_{0}, \quad \tilde{v}^{\rho} \in \mathscr{U}_{0}, \quad \frac{d}{d t}\left(A \tilde{\sigma}^{\rho}\right)+\partial \tilde{\varphi}\left(t, \tilde{\sigma}^{\rho}\right)=\varepsilon\left(\tilde{v}^{\rho}\right), \\
\rho \frac{d \tilde{v}^{\rho}}{d t}-D \tilde{\sigma}^{\rho}=-\rho \frac{d v_{s}^{\mathrm{el}}}{d t}, \quad \tilde{\sigma}^{\rho}(0)=0, \quad \tilde{v}^{\rho}(0)=v_{0}-v_{s}^{\mathrm{el}}(0) .
\end{gathered}
$$

A priori estimates I. Take $\tilde{\sigma}^{\rho}$ and $\tilde{v}^{\rho}$ as test functions in (4.1). After integration we get:

$$
\begin{aligned}
\alpha\left|\tilde{\sigma}^{\rho}(t)\right|^{2} & +\frac{\rho}{2}\left|\tilde{v}^{\rho}(t)-\tilde{v}^{\rho}(0)\right|^{2}+\int_{0}^{t}\left(\partial \tilde{\varphi}\left(s, \tilde{\sigma}^{\rho}(s)\right), \tilde{\sigma}^{\rho}(s)\right) d s \\
& \leq \rho\left|\tilde{v}^{\rho}(0)\right| \int_{0}^{t}\left|\tilde{v}^{\rho}(s)\right| d s+\int_{0}^{t}\left|\frac{d A}{d t}\right|\left|\tilde{\sigma}^{\rho}(s)\right|^{2} d s+\int_{0}^{t} \rho\left|\frac{d v_{s}^{\mathrm{el}}}{d t}\right|\left|\tilde{v}^{\rho}\right| d s .
\end{aligned}
$$

But

$$
\int_{0}^{t}\left(\partial \tilde{\varphi}\left(s, \tilde{\sigma}^{\rho}\right), \tilde{\sigma}^{\rho}\right) \geq \int_{0}^{t} \varphi\left(s, \sigma^{\rho}\right) d s-\int_{0}^{t} \varphi\left(\theta, \sigma_{s}^{\mathrm{el}}\right) d \theta .
$$

Then by the Gronwall lemma, we get:

$$
\begin{aligned}
& \tilde{\sigma}^{\rho} \text { is a bounded sequence in } L^{\infty}(0, T ; H), \\
& \rho^{1 / 2} \tilde{v}^{\rho} \text { is a bounded sequence in } L^{\infty}(0, T ; V), \\
& \int_{0}^{t} \varphi\left(s, \sigma^{\rho}(s)\right) d s \leq c .
\end{aligned}
$$

A priori estimates II. We write (4.1) at times $s+h$ and $s$, and we take the difference: choosing $\delta_{h} \tilde{\sigma}^{\rho}(s)=\left(\tilde{\sigma}^{\rho}(s+h)-\tilde{\sigma}^{\rho}(s)\right) / h, \quad \delta_{h} \tilde{v}^{\rho}(s)$ as test functions, we get after 
integration:

$$
\begin{aligned}
& \alpha\left|\delta_{h} \tilde{\sigma}^{\rho}(t)\right|^{2}+\frac{\rho}{2}\left|\delta_{h} \tilde{v}^{\rho}(t)\right|^{2}+\int_{0}^{t}\left(\delta_{h} \partial \tilde{\varphi}\left(s, \tilde{\sigma}^{\rho}(s)\right), \tilde{\sigma}^{\rho}(s)\right) d s \\
& \quad \leq \alpha\left|\delta_{h} \tilde{\sigma}^{\rho}(0)\right|^{2}+\frac{\rho}{2}\left|\delta_{h} \tilde{v}^{\rho}(0)\right|^{2}+\int_{0}^{t}\left|\frac{d A}{d t}\right|\left|\delta_{h} \tilde{\sigma}^{\rho}\right|^{2} d s+\int_{0}^{t} c \rho\left|\delta_{h} \tilde{v}^{\rho}\right| d s .
\end{aligned}
$$

But by monotonicity:

$$
\int_{0}^{t}\left(\delta_{h} \partial \tilde{\varphi}\left(s, \tilde{\sigma}^{\rho}\right), \tilde{\sigma}^{\rho}\right) d s \geq \int_{0}^{t}\left(\frac{\partial \varphi\left(s+h, \sigma^{\rho}\right)-\partial \varphi\left(s, \sigma^{\rho}\right)}{h}, \delta_{h} \tilde{\sigma}^{\rho}\right) d s .
$$

It follows from (4.2) that

$$
\left|\delta_{h} \tilde{\sigma}^{\rho}(0)\right|^{2} \leq c, \quad \rho\left|\delta_{h} \tilde{v}^{\rho}(0)\right|^{2} \leq c .
$$

Then, using (4.6), (4.7) and assumption (2.9), we get:

$$
\begin{aligned}
\alpha\left|\delta_{h} \tilde{\sigma}^{\rho}(t)\right|^{2}+\frac{\rho}{2}\left|\delta_{h} \tilde{v}^{\rho}(t)\right|^{2} \leq & c+c \int_{0}^{t}\left(c+\varphi\left(s, \sigma^{\rho}(s)\right)\right)\left|\delta_{h} \tilde{\sigma}^{\rho}(s)\right| d s \\
& +c_{3} \int_{0}^{t}\left|\delta_{h} \tilde{\sigma}^{\rho}\right|^{2} d s+c_{4} \int_{0}^{t} \rho\left|\delta_{h} \tilde{v}^{\rho}\right| d s .
\end{aligned}
$$

By the Gronwall lemma and the estimate (4.5) we establish:

$\delta_{h} \tilde{\sigma}^{\rho} \quad$ is a bounded sequence in $L^{\infty}(0, T ; H)$ independently of $h$. $\rho^{1 / 2} \delta_{h} \tilde{v}^{\rho}$ is a bounded sequence in $L^{\infty}(0, T ; V)$ independently of $h$.

We let $h$ tend to 0 and we obtain:

$$
\begin{gathered}
\frac{d \tilde{\sigma}^{\rho}}{d t} \text { is a bounded sequence in } L^{\infty}(0, T ; H), \\
\rho^{1 / 2} \frac{d \tilde{v}^{\rho}}{d t} \quad \text { is a bounded sequence in } L^{\infty}(0, T ; V) .
\end{gathered}
$$

Due to the regularity assumption on $\left(\sigma_{s}^{\mathrm{el}}, v_{s}^{\mathrm{el}}\right)$ we get the same estimates for $d \sigma^{\rho} / d t$ and $\rho^{1 / 2}\left(d v^{\rho} / d t\right)$. Coming back to (4.1), we see that

$$
\left(\partial \tilde{\varphi}\left(t, \tilde{\sigma}^{\rho}\right), \tilde{\sigma}^{\rho}\right)=-\left(\frac{d\left(A(t) \tilde{\sigma}^{\rho}\right)}{d t}, \tilde{\sigma}^{\rho}\right)-\rho\left(\frac{d \tilde{v}^{\rho}}{d t}, \tilde{v}^{\rho}\right)-\rho\left(\frac{d v_{s}^{\mathrm{el}}}{d t}, \tilde{v}^{\rho}\right) ;
$$

hence $\left(\partial \tilde{\varphi}\left(t, \tilde{\sigma}^{\rho}\right), \tilde{\sigma}^{\rho}\right)$ is bounded in $L^{\infty}(0, T)$. But

$$
\varphi(t, \sigma)=\tilde{\varphi}\left(t, \tilde{\sigma}^{\rho}\right) \leq \tilde{\varphi}(t, 0)+\left(\partial \tilde{\varphi}\left(t, \tilde{\sigma}^{\rho}\right), \tilde{\sigma}^{\rho}\right) \leq c
$$

and it follows from assumption (2.7) and estimate (4.3) that:

$$
\sigma^{\rho} \text { is a bounded sequence in } L^{\infty}(0, T ; X),
$$

and by assumption $(2.8)$ :

$$
\partial \varphi\left(\cdot, \sigma^{\rho}\right) \text { is a bounded sequence in } L^{\infty}\left(0, T ; X^{\prime}\right) .
$$


(4.8) and (4.11) together with (4.1) show that $\varepsilon\left(\tilde{v}^{\rho}\right)$ is bounded in $L^{\infty}\left(0, T ; X^{\prime}\right)$, and (4.9) does the same for $D \tilde{\sigma}^{\rho}$ in $L^{\infty}(0, T ; V)$. Then

$\tilde{v}^{\rho} \quad$ is a bounded sequence in $L^{\infty}\left(0, T ; \mathscr{U}_{0}\right) \quad\left(\right.$ since $\left.\partial \Omega_{U} \neq \phi\right)$,

$\tilde{\sigma}^{\rho} \quad$ is a bounded sequence in $L^{\infty}\left(0, T ; \mathscr{H}_{0}\right)$.

Then there exists $(\tilde{\sigma}, \chi, \tilde{v}) \in L^{\infty}\left(0, T ; \mathscr{H}_{0}\right) \times L^{\infty}\left(0, T ; X^{\prime}\right) \times L^{\infty}\left(0, T ; \mathscr{U}_{0}\right)$ such that:

$$
\begin{array}{ccc}
\tilde{\sigma}^{\rho}-\tilde{\sigma} & \text { in } & L^{\infty}\left(0, T ; \mathscr{H}_{0}\right) \text { weak*, } \\
\tilde{v}^{\rho}-\tilde{v} & \text { in } & L^{\infty}\left(0, T ; \mathscr{U}_{0}\right) \text { weak*, } \\
d \tilde{\sigma}^{\rho} / d t-d \tilde{\sigma} / d t & \text { in } & L^{\infty}(0, T ; H) \text { weak*, } \\
\partial \varphi\left(t, \sigma^{\rho}\right)-\chi & \text { in } & L^{\infty}\left(0, T ; X^{\prime}\right) \text { weak*. }
\end{array}
$$

It can be shown that $\tilde{\sigma}^{\rho}$ tends to $\tilde{\sigma}$ in $L^{\infty}(0, T ; H)$ strongly:

$$
\left(\frac{d A \tilde{\sigma}^{\rho}}{d t}-\frac{d A \tilde{\sigma}^{\rho^{\prime}}}{d t}, \tilde{\sigma}^{\rho}-\tilde{\sigma}^{\rho^{\prime}}\right)+\rho\left(\frac{d \tilde{v}^{\rho}}{d t}, \tilde{v}^{\rho}-\tilde{v}^{\rho^{\prime}}\right)-\rho^{\prime}\left(\frac{d \tilde{v}^{\rho^{\prime}}}{d t}, \tilde{v}^{\rho}-\tilde{v}^{\rho^{\prime}}\right) \leq 0 .
$$

Then by integration:

$$
\begin{aligned}
\alpha\left|\tilde{\sigma}^{\rho}(t)-\tilde{\sigma}^{\rho^{\prime}}(t)\right|^{2}+ & \frac{\rho}{2}\left|\tilde{v}^{\rho}(t)-\tilde{v}^{\rho^{\prime}}(t)\right|^{2} \\
& \leq\left|\rho-\rho^{\prime}\right| \int_{0}^{t}\left|\frac{d \tilde{v}^{\rho^{\prime}}}{d t}\right|\left|\tilde{v}^{\rho}-\tilde{v}^{\rho^{\prime}}\right| d s+c \int_{0}^{t}\left|\tilde{\sigma}^{\rho}(s)-\tilde{\sigma}^{\rho^{\prime}}(s)\right|^{2} d s .
\end{aligned}
$$

Applying estimate (4.9):

$$
\left|\tilde{\sigma}^{\rho}(t)-\tilde{\sigma}^{\rho^{\prime}}(t)\right|^{2} \leq c_{1}\left|\rho-\rho^{\prime}\right|^{1 / 2}+c_{2} \int_{0}^{t}\left|\tilde{\sigma}^{\rho}(s)-\tilde{\sigma}^{\rho^{\prime}}(s)\right|^{2} d s,
$$

and by the Gronwall lemma:

$$
\left|\tilde{\sigma}^{\rho}(t)-\tilde{\sigma}^{\rho^{\prime}}(t)\right|^{2} \leq c_{1}\left|\rho-\rho^{\prime}\right|^{1 / 2} e^{c_{2} t}
$$

which proves that $\tilde{\sigma}^{\rho}$ is a Cauchy sequence in $L^{\infty}(0, T ; H)$. Then $\tilde{\sigma}$ satisfies $\tilde{\sigma}(0)=$ $\lim _{\rho \rightarrow 0} \tilde{\sigma}^{\rho}(0)=0$. It can easily be shown that $(\tilde{\sigma}, \chi, \tilde{v})$ is solution of:

$$
\frac{d(A \tilde{\sigma})}{d t}+\chi=\varepsilon(\tilde{v}), \quad-D \tilde{\sigma}=0, \quad \tilde{\sigma}(0)=0 .
$$

It remains to show that $\chi(t)=\partial \varphi(t, \sigma(t))$. This is done by a monotonicity argument: it is sufficient to establish:

$$
\int_{0}^{T}(\chi(t)-\partial \varphi(t, \tau(t)), \sigma(t)-\tau(t)) d t \geq 0 \quad\left(\forall \tau \in L^{\infty}(0, T ; X)\right.
$$

But

$$
\int_{0}^{T}\left(\partial \varphi\left(t, \sigma^{\rho}(t)\right)-\partial \varphi(t, \tau(t)), \sigma^{\rho}(t)-\tau(t)\right) d t \geq 0
$$


and the difficult point is to show that

$$
\int_{0}^{T}(\chi(t), \sigma(t)) d t \geq \limsup _{\rho \rightarrow 0} \int_{0}^{T}\left(\partial \varphi^{\rho}\left(t, \sigma^{\rho}(t)\right), \sigma^{\rho}(t)\right) d t
$$

But

$$
\begin{aligned}
\int_{0}^{T}\left(\partial \varphi\left(t, \sigma^{\rho}\right), \sigma^{\rho}\right) d t= & -\int_{0}^{T}\left[\left(\frac{d A \tilde{\sigma}^{\rho}}{d t}, \tilde{\sigma}^{\rho}\right)+\rho\left(\frac{d \tilde{v}^{\rho}}{d t}, \tilde{v}^{\rho}\right)\right. \\
& \left.+\rho\left(\frac{d v_{s}^{\mathrm{el}}}{d t}, \tilde{v}^{\rho}\right)+\left(\partial \varphi\left(t, \sigma^{\rho}\right), \sigma_{s}^{\mathrm{el}}\right)\right] d t .
\end{aligned}
$$

By the monotonicity assumption (Remark (3.2)) we get:

$$
\int_{0}^{T}\left(\frac{d A \tilde{\sigma}}{d t}, \tilde{\sigma}\right) d t \leq \liminf _{\rho \rightarrow 0} \int_{0}^{T}\left(\frac{d A \tilde{\sigma}^{\rho}}{d t}, \tilde{\sigma}^{\rho}\right) d t
$$

which proves (4.15) and ends the proof of Proposition 2.

5. The perfectly plastic problem. The perfectly plastic constitutive law is obtained when $\varphi$ is the indicator function of the closed convex set of elasticity. In order to formulate the problem, we need some more notation:

$$
\begin{aligned}
\mathscr{P}(t) & =\{\tau \mid \tau \in H, \tau(x) \in B(x, t) \text { a.e. in } \Omega\}, \\
S(t) & =\{\tau \mid \tau \in-D \tau=f(t) \text { a.e. in } \Omega\}, \\
B D(\Omega) & =\left\{u \mid u=\left(u_{i}\right) ; u_{i} \in L^{1}(\Omega) ; \varepsilon_{i j}(u) \in M^{1}(\Omega)\right\},
\end{aligned}
$$

where $M^{1}(\Omega)$ is the space of bounded measures on $\Omega$. This space has been introduced simultaneously and independently in [20] and [28], and has been studied in [27] and [29]. Some of its properties are recalled in the appendix. An important result, due to Matthies-Strang-Christiansen [20], is that $B D(\Omega)$ is the dual of a Banach space. However, $B D(\Omega)$ is a non-reflexive, non-separable space.

$L^{p}(0, T ; Z)$ denotes the space of measurable functions $f:[0, T] \rightarrow Z$ such that:

$$
\int_{0}^{T}\|f(t)\|_{Z}^{p} d t<+\infty
$$

Its dual space $L_{w}^{p^{\prime}}\left(0, T ; Z^{\prime}\right)$ is composed of weakly measurable functions $g:[0, T] \rightarrow Z^{\prime}$ in the following sense: $t \rightarrow\langle g(t), z\rangle_{Z, Z^{\prime}}$ is a measurable function for every $z \in Z$ which moreover satisfy $\int_{0}^{T}\|g(t)\|_{Z^{\prime}}^{p^{\prime}} d t<+\infty$ (see [8]). When $Z^{\prime}$ is a reflexive or a separable space, $L_{w}^{p^{\prime}}\left(0, T ; Z^{\prime}\right)=L^{p^{\prime}}\left(0, T ; Z^{\prime}\right)$.

Formulation of the problem. The quasi-static perfectly plastic problem is more difficult to formulate than the regular problems. This difficulty is due to the non-regularity of the strain tensor. A strong formulation analogous to (2.2) would be: find $(\sigma, v) \times$ $[0, T] \rightarrow \mathscr{P}(t) \cap \mathscr{H}_{\text {ad }}(t) \times \mathscr{V}_{\text {ad }}$ such that:

$$
\begin{array}{crrl}
(d(A \sigma) / d t, \tau-\sigma) & \geq(\varepsilon(v), \tau-\sigma) & & (\forall \tau \in \mathscr{P}(t)), \\
-D \sigma=f, & \sigma(0)=\sigma_{0}, \quad v=\dot{u}, & u(0)=u_{0} .
\end{array}
$$


Unfortunately, this strong formulation has no solution $v \in \mathscr{V}_{\text {ad }}$ in the general case; the physical solutions have not the regularity required by $\mathscr{V}_{\text {ad }}$, since they can be discontinuous. (Elements of $\mathscr{V}_{\text {ad }}$ admit a trace on every surface included in $\Omega$.) As in shock theory, the classical formulation cannot account for these discontinuities, and a notion of weak solution must be introduced. This is done by mean of Green's formula:

$$
(\varepsilon(v), \tau-\sigma)+(v, D \tau-D \sigma)=\int_{\partial \Omega u} \dot{U}_{i}^{d}\left(\tau_{i j}-\sigma_{i j}\right) n_{j} d s
$$

where $\tau$ and $\sigma$ belong to $\mathscr{H}_{\text {ad }}$. The weak formulation is the following: find $(\sigma, v):[0, T] \rightarrow$ $\mathscr{P}(t) \cap \mathscr{H}_{\text {ad }} \times B D(\Omega)$ such that:

$$
\begin{gathered}
\left(\frac{d(A \sigma)}{d t}, \tau-\sigma\right)+(v, D \tau-D \sigma) \geq \int_{\partial \Omega U} \dot{U}_{i}^{d}\left(\tau_{i j}-\sigma_{i j}\right) n_{j} d s, \text { for } \tau \in \mathscr{P}(t) \cap \mathscr{H}_{\mathrm{ad}}(t), \\
-D \sigma=f, \quad \sigma(0)=\sigma_{0}
\end{gathered}
$$

Note that the boundary conditions on $v$ (or $u$ ) are included in the first line of (5.3).

Safety assumptions. In addition to the assumptions made in the regular case, we shall make a further hypothesis. In order to obtain a solution $\sigma \in S(t)$, we must at least assume that $S(t)$ is non-empty. We make the following stronger assumption, already introduced by Moreau [22] and Johnson [9]: there exists $\chi(t)$ defined on $[0, T]$ such that:

$$
\begin{gathered}
\chi(t) \in S(t) \quad \text { a.e. in }[0, T], \\
\exists \delta>0: \chi(t)+\tau \in \mathscr{P}(t) \quad \text { a.e. in }[0, T], \quad \forall \tau \in\left(L^{\infty}(\Omega)\right)_{s}^{9}:\|\tau\|_{\infty} \leq \delta, \\
\chi \in L^{\infty}\left(0, T ;\left(L^{\infty}(\Omega)\right)_{s}^{9}\right), \quad \partial \chi / \partial t \in L^{\infty}\left(0, T ;\left(L^{\infty}(\Omega)\right)_{s}^{9}\right) .
\end{gathered}
$$

We also assume that 0 is in the interior of $\mathscr{P}(t)$ (uniformly on $t$ ).

Then the problem (5.3) can be solved via a sequence of regularized problems, the potentials of which satisfy:

$$
\tau \in \mathscr{P}(t) \Leftrightarrow \lim _{n \rightarrow+\infty} \varphi^{n}(t, \tau)=0,
$$

If there exists some constant $C$ such that $\varphi^{n}(t, \tau)=0$

$$
\text { then } \lim _{n \rightarrow+\infty} \varphi^{n}(t, \tau)=0 \text { and } \tau \in \mathscr{P}(t) .
$$

It can be easily shown that (5.6) and (5.7) are satisfied for the Perzyna potential (with $n=1 / \mu$ ) and for the Norton-Hoff potential (with $n=p$ ).

THEOREM. Under the above assumptions there exists a solution $(\sigma, v)$ of $(5.3)$, unique in $\sigma$, such that $\sigma \in L^{\infty}(0, T ; H), \dot{\sigma} \in L^{2}(0, T ; H), v \in L_{w}^{2}(0, T ; B D(\Omega))$. Moreover:

$$
\begin{aligned}
& \sigma^{n}-\sigma \quad \text { in } \quad L^{\infty}(0, T ; H) \text { weak* }^{*}, \\
& \dot{\sigma}^{n} \rightarrow \dot{\sigma} \quad \text { in } \quad L^{\infty}(0, T ; H) \text { weak* }^{*}, \\
& v^{n} \rightarrow v \quad \text { in } \quad L_{w}^{2}(0, T ; B D(\Omega)) \text { weak* }^{*} .
\end{aligned}
$$


Proof. We consider a sequence of potentials $\varphi^{n}$ satisfying (5.6), (5.7), and we denote by $\left(\sigma^{n}, v^{n}\right)$ the unique solutions of the corresponding regular problems:

$$
\frac{d A \sigma^{n}}{d t}+\partial \varphi^{n}\left(t, \sigma^{n}\right)=\varepsilon\left(v^{n}\right), \quad-D \sigma^{n}=f, \quad \sigma^{n}(0)=\sigma_{0} .
$$

A priori estimates I. Set $\bar{\sigma}^{n}=\sigma^{n}-\chi$. Taking as test functions $\bar{\sigma}^{n}$ and $v^{n}$, we get

$$
\left(\frac{d\left(A \bar{\sigma}^{n}\right)}{d t}, \bar{\sigma}^{n}\right)+\left(\partial \varphi^{n}\left(t, \sigma^{n}\right), \bar{\sigma}^{n}\right)=\left(\varepsilon\left(v^{n}\right), \bar{\sigma}^{n}\right)+\left(v^{n}, D \bar{\sigma}^{n}\right)
$$

But

$$
\left(\varepsilon\left(v^{n}\right), \bar{\sigma}^{n}\right)+\left(v^{n}, D \bar{\sigma}^{n}\right)=\left(\varepsilon\left(v_{s}^{\mathrm{el}}\right), \bar{\sigma}^{n}\right),
$$

and

$$
\left(\partial \varphi^{n}\left(t, \sigma^{n}\right), \tilde{\sigma}^{n}\right) \geq \varphi^{n}\left(t, \sigma^{n}\right)-\varphi^{n}(t, \chi)
$$

By integration:

$$
\alpha\left|\bar{\sigma}^{n}(\tau)\right|^{2}+\int_{0}^{T} \varphi^{n}\left(\theta, \sigma^{n}\right) d \theta \leq \int_{0}^{T} \varphi^{n}(\theta, \chi) d \theta+\int_{0}^{T}\left[\left(\varepsilon\left(v_{s}^{\mathrm{el}}\right), \bar{\sigma}^{n}\right)+\left|\frac{d A}{d t}\right|\left|\bar{\sigma}^{n}\right|^{2} \mid d t .\right.
$$

It follows from the Gronwall lemma and the regularity assumptions that:

$\bar{\sigma}^{n}\left(\right.$ and $\left.\sigma^{n}\right)$ is a bounded sequence in $L^{\infty}(0, T ; H)$,

$$
\int_{0}^{T} \varphi^{n}\left(\theta, \sigma^{n}\right) d \theta \leq c
$$

Multiplying (5.8) by $\bar{\sigma}^{n}$ and integrating, we get:

$$
\int_{0}^{T}\left(\partial \varphi^{n}\left(\theta, \sigma^{n}\right), \bar{\sigma}^{n}\right) d \theta \leq c
$$

Let $\tau \in L^{\infty}\left(0, T ;\left(L^{\infty}(\Omega)\right)_{s}^{9}\right)$ such that $\|\tau(t)\|_{\infty} \leq \delta$ a.e. in [0,T]. By assumption (5.4) $\chi+\tau \in \mathscr{P}(t)$ a.e. in $[0, T]$ and:

$$
\int_{0}^{T}\left(\partial \varphi^{n}\left(t, \sigma^{n}\right), \tau\right) d t \leq \int_{0}^{T}\left(\partial \varphi^{n}\left(t, \sigma^{n}\right), \bar{\sigma}^{n}\right) d t+\int_{0}^{T} \varphi^{n}(\chi+\tau) d t-\int_{0}^{T} \varphi^{n}\left(\sigma^{n}\right) d t .
$$

We conclude, by means of estimate (5.10) and assumption (2.7), that for every $\tau \in L^{\infty}\left(0, T ;\left(L^{\infty}(\Omega)\right)_{s}^{9}\right),\|\tau(t)\|_{\infty} \leq \delta$, we have

$$
\int_{0}^{T}\left(\partial \varphi^{n}\left(t, \sigma^{n}\right), \tau\right) d t \leq c, \text { i.e., } \partial \varphi^{n}\left(\cdot, \sigma^{n}\right) \text { is bounded in } L^{1}\left(0, T ;\left(L^{1}(\Omega)\right)_{s}^{9}\right)
$$

A priori estimates II. We now take $d \bar{\sigma}^{n} / d t$ as test function. Then

$$
\begin{gathered}
\left(\frac{d A \bar{\sigma}^{n}}{d t} \frac{d \bar{\sigma}^{n}}{d t}\right)+\left(\partial \varphi^{n}\left(t, \sigma^{n}\right), \frac{d \bar{\sigma}^{n}}{d t}\right)=\left(\varepsilon\left(v^{n}\right), \frac{d \bar{\sigma}^{n}}{d t}\right)+\left(\frac{d A \chi}{d t}, \frac{d \bar{\sigma}^{n}}{d t}\right), \\
\left(\varepsilon\left(v^{n}\right), \frac{d \bar{\sigma}^{n}}{d t}\right)=\left(\varepsilon\left(v_{s}^{\mathrm{el}}\right), \frac{d \bar{\sigma}^{n}}{d t}\right), \\
\left(\partial \varphi^{n}\left(t, \sigma^{n}\right), \frac{d \bar{\sigma}^{n}}{d t}\right)=\frac{d}{d t}\left[\varphi^{n}\left(t, \sigma^{n}\right)\right]-\frac{\partial \varphi^{n}}{\partial t}\left(t, \sigma^{n}\right)-\left(\partial \varphi^{n}\left(t, \sigma^{n}\right), \frac{\partial \chi}{\partial t}\right),
\end{gathered}
$$


so that integration yields

$$
\begin{aligned}
\alpha \int_{0}^{T}\left|\frac{d \bar{\sigma}^{n}}{d t}\right|^{2} d t+\varphi^{n}\left(T, \sigma^{n}(T)\right) \leq \varphi^{n}\left(0, \sigma_{0}\right)+ & \int_{0}^{T}\left|\left(\varepsilon\left(v_{s}^{\mathrm{el}}\right), \frac{d \bar{\sigma}^{n}}{d t}\right)+\right| \frac{d A \chi}{d t}|| \frac{d \bar{\sigma}^{n}}{d t}|| d t \\
& +\int_{0}^{T}\left|\frac{\partial \varphi^{n}}{\partial t}\left(t, \sigma^{n}\right)\right| d t+\int_{0}^{T}\left(\partial \varphi^{n}\left(t, \sigma^{n}\right), \frac{d \chi}{d t}\right) d t .
\end{aligned}
$$

From (5.12) and assumption (2.9), the last term of (5.13) is bounded. We conclude that:

$$
\frac{d \bar{\sigma}^{n}}{d t}\left(\text { and } \frac{d \sigma^{n}}{d t}\right) \text { is a bounded sequence in } L^{2}(0, T ; H) \text {. }
$$

Taking $\bar{\sigma}^{n}$ as test function in (5.8), we get:

$$
\left(\partial \varphi^{n}\left(t, \sigma^{n}\right), \bar{\sigma}^{n}\right)=-\left(\frac{d A \sigma^{n}}{d t}, \bar{\sigma}^{n}\right)+\left(\varepsilon\left(v^{\mathrm{el}}\right), \bar{\sigma}^{n}\right) .
$$

$d A \sigma^{n} / d t$ and $\bar{\sigma}^{n}$ are respectively bounded in $L^{2}(0, T ; H)$ and $L^{\infty}(0, T ; H)$. Then $\left(\partial \varphi^{n}\left(t, \sigma^{n}\right), \bar{\sigma}^{n}\right)$ is bounded in $L^{2}(0, T)$. By the same reasoning as above we show:

$$
\partial \varphi^{n}\left(t, \sigma^{n}\right) \text { is a bounded sequence in } L^{2}\left(0, T ;\left(L^{1}(\Omega)\right)_{s}^{9}\right)
$$

and by, (5.8),

$$
\varepsilon\left(v^{n}\right) \quad \text { is a bounded sequence in } L^{2}\left(0, T ;\left(L^{1}(\Omega)\right)_{s}^{9}\right)
$$

It results from a theorem of equivalent norms ([27] or [28]), that due to the boundary conditions:

$$
v^{n} \quad \text { is a bounded sequence in } L^{2}(0, T ; B D(\Omega)) \text {. }
$$

We know ([18]) that $B D(\Omega)$ is the dual space of a Banach space $C$. Then $L_{w}^{2}(0, T ; B D(\Omega))$ is the dual of $L^{2}(0, T ; C)$, and we deduce from (5.15), (5.9), (5.11) that there exist $\sigma \in W^{1,2}(0, T ; H), v \in L_{w}^{2}(0, T ; B D(\Omega))$ such that:

$$
\begin{gathered}
\sigma^{n} \rightarrow \sigma \quad \text { in } \quad L^{\infty}(0, T ; H) \text { weak*, } \\
\dot{\sigma}^{n} \rightarrow \dot{\sigma} \quad \text { in } \quad L^{2}(0, T ; H) \text { weak, } \\
v^{n} \rightarrow v \quad \text { in } \quad L_{w}^{2}(0, T ; B D(\Omega)) \text { weak }^{*} .
\end{gathered}
$$

It is clear from (5.17) that $d A \sigma^{n} / d t \rightarrow d A \sigma / d t$ in $L^{2}(0, T ; H)$ weak and that $\sigma(0)=\sigma_{0}$, - $D \sigma=f$. We multiply (5.8) by $\tau-\sigma^{n}$, where $\tau(t) \in \mathscr{P}(t) \cap \mathscr{H}_{\text {ad }}(t)$, and integrate:

$$
\begin{aligned}
\int_{0}^{T}\left(\frac{d A \sigma^{n}}{d t}, \tau-\sigma^{n}\right) d t+\int_{0}^{T}\left(\partial \varphi^{n}\left(t, \sigma^{n}\right), \tau\right. & \left.-\sigma^{n}\right) d t+\int_{0}^{T}\left(v^{n}, D \tau-D \sigma^{n}\right) d t \\
& \geq \int_{0}^{T} d t \int_{i \Omega v} \dot{U}_{i}^{d}\left(\tau_{i j}-\sigma_{i j}\right) n j, \quad-D \sigma^{n}=f .
\end{aligned}
$$

By Remark 3.2, the operator

$$
\sigma \rightarrow \int_{0}^{T}\left(\frac{d A \sigma}{d t}(t), \sigma(t)\right) d t
$$


is monotone. Hence

$$
\lim _{n \rightarrow+\infty} \int_{0}^{T}\left(\frac{d A \sigma^{n}}{d t}, \sigma^{n}\right) d t \geq \int_{0}^{T}\left(\frac{d(A \sigma)}{d t}, \sigma\right) d t
$$

and

$$
\int_{0}^{T}\left(\frac{d A \sigma}{d t}, \tau-\sigma\right) d t \geq \limsup _{n \rightarrow+\infty} \int_{0}^{T}\left(\frac{d A \sigma^{n}}{d t}, \tau-\sigma^{n}\right) d t
$$

Furthermore,

$$
\lim _{n \rightarrow+\infty} \int_{0}^{T}\left(v^{n}, D \tau-D \sigma^{n}\right) d t=\int_{0}^{T}(v, D \tau-D \sigma) d t
$$

(since $-D \sigma^{n}=f$ ), and

$$
\left(\partial \varphi^{n}\left(t, \sigma^{n}\right), \tau-\sigma^{n}\right) \leq \varphi^{n}(t, \tau)-\varphi^{n}\left(t, \sigma^{n}\right) \leq \varphi^{n}(t, \tau) .
$$

Then, using assumption (5.6), $\lim _{n \rightarrow+\infty}\left(\partial \varphi^{n}\left(t, \sigma^{n}\right), \tau-\sigma^{n}\right)=0$, and we have shown that:

$$
\int_{0}^{T}\left(\frac{d A \sigma}{d t}, \tau-\sigma\right) d t+\int_{0}^{T}(v, D \tau-D \sigma) d t \geq \int_{0}^{T} d t \int_{\tau \Omega U} \dot{U}_{i}^{d}\left(\tau_{i j}-\sigma_{i j}\right) n j d S,
$$

which proves $(5.3)$ by a classical use of Lebesgue points for a $L^{1}$ function. It follows from (5.10) and assumption (5.7) that $\sigma(t)$ belongs to $\mathscr{P}(t)$.

Uniqueness of $\sigma$. If there exist two solutions $(\sigma, v)$ and $\left(\sigma^{*}, v^{*}\right)$ they must satisfy:

$$
\left(\frac{d\left(A \sigma-A \sigma^{*}\right)}{d t}, \sigma-\sigma^{*}\right) \leq 0
$$

Hence

$$
\alpha\left|\sigma-\sigma^{*}\right|^{2}(t) \leq \alpha\left|\sigma-\sigma^{*}\right|^{2}(0)+\int_{0}^{t}\left|\frac{d A}{d t}\right|\left|\sigma-\sigma^{*}\right|^{2}(s) d s,
$$

and by the Gronwall lemma:

$$
\left|\sigma-\sigma^{*}\right|^{2}(t) \leq\left|\sigma-\sigma^{*}\right|^{2}(0) e^{c t}=0 .
$$

Remark 5.1. We are not able to prove the uniqueness of $v$, and this result seems to be false.

Remark 5.2. The trace theorem for $B D(\Omega)$ recalled in the appendix indicates the regularity of the solution $v$; this velocity field can be discontinuous on every surface included in $\Omega$. No systematic investigation of these surfaces of discontinuity has been performed in connection with the work of Volpert [31] on $B V(\Omega)$ (part of this has been done by Kohn [13].

7. The boundary conditions. The boundary conditions in velocity are contained in the weak formulation of the problem. A trace theorem has been established for $B D(\Omega)$ which gives a sense to $v$ on $\partial \Omega$. One could expect that in this sense the boundary conditions $v_{i}=U_{i}^{d}$ are satisfied on $\partial \Omega_{U}$. This is not true since a slip phenomenon can occur on $\partial \Omega_{U}$; the boundary conditions are satisfied by the external trace of the velocity, but not necessarily by the internal trace. The dissipation due to the discontinuities is not 
negligible and must be taken as part of the total dissipation of the plastic body. Hence, a plasticity problem must be considered not only as a classical boundary-value problem on the open set, but at least as a problem on the set $\bar{\Omega}$.

Mathematical interpretation. We define on $B D\left(R^{N}\right)$ an equivalence relation:

$$
u \mathscr{R} v \Leftrightarrow\left\{\begin{array}{c}
\left.u\right|_{\Omega}=\left.v\right|_{\Omega} \\
\left.\varepsilon_{i j}(u)\right|_{\bar{\Omega}}=\left.\varepsilon_{i j}(v)\right|_{\bar{\Omega}} \quad \text { in } \quad M(\bar{\Omega})
\end{array} .\right.
$$

Then it can be checked that the external traces $u^{+}$and $v^{+}$on $\partial \Omega$ (in the sense defined in the appendix) are equal. We set

$$
\begin{aligned}
B D(\bar{\Omega}) & =B D\left(R^{N}\right) / \mathscr{R}, \\
B D_{\mathrm{ad}}(\bar{\Omega}) & =\left\{v \mid v \in B D(\bar{\Omega}), v_{i}^{+}=\dot{U}_{i}^{d} \text { on } \partial \Omega_{u}\right\} .
\end{aligned}
$$

It can be proved that the solution $v$ of the theorem can be extended in an element of $B D_{\mathrm{ad}}(\bar{\Omega})$; it suffices to extend into $R^{N}$ the solutions $v^{n}$ of the regularized problems and to take the limit of these extensions. After quotient by the equivalence relation $\mathscr{R}$ the limit belongs to $B D_{\mathrm{ad}}(\bar{\Omega})$ and does not depend on the chosen extension.

This problem of unsatisfied boundary conditions is usual in the theory of minimal surfaces (where the solution is in $B V(\Omega)$ ). Using this analogy, Temam [30] has proposed to relax the boundary conditions. This procedure is equivalent to ours, but is more convenient from a mathematical point of view for the study of Hencky's material. We believe that it is also the case for the Prandtl-Reuss material studied in this paper. Recent works on minimal surfaces assert that the boundary conditions are satisfied on the parts of the boundary with positive curvature (see [14]).

8. Conclusion. We have tried to give a mathematical form for the theory of a large class of dissipative materials, often used for numerical computations. We have pointed out that in the case of a regular potential of dissipation the velocity solutions for these materials are regular. On the contrary, for strongly dissipative materials such as perfectly plastic bodies the velocity solutions can be discontinuous, even on the boundary. The approximation of the perfectly plastic problem by regularized models has been justified.

Appendix. The following trace theorem, extending a result of Miranda on $B V(\Omega)$, has been proved by Strang and Temam [27] and the author [29].

THEOREM 2. Let $\Omega$ be a regular open set of $R^{N}$. There exists a surjective and linear application $\gamma_{-}$from $B D(\Omega)$ to $\left(L^{1}(\partial \Omega)\right)^{N}$ such that for every $\varphi \in(\mathscr{D}(\bar{\Omega}))_{s}^{N^{2}}$ we have:

$$
\int_{\Omega} u_{i} \varphi_{i j, j} d x+\int_{\Omega} \varphi_{i j} d \varepsilon_{i j}(u)=\int_{i \Omega}(\gamma-u)_{i} \varphi_{i j} n j d s \quad(\forall u \in B D(\Omega)) .
$$

$u^{-}=\gamma_{-} u$ is called the internal trace of $u$ on $\partial \Omega$. When $u$ is a regular function $\left(C^{0}(\bar{\Omega})\right) u^{-}$ is the classical trace of $u$ on $\partial \Omega$. Moreover, if $(S)$ is a locally $(N-1)$-dimensional manifold included in $\Omega$, dividing $\Omega$ into two non-empty components $\Omega_{-}$and $\Omega_{+}$, there 
exist two surjective maps $\gamma_{-}$and $\gamma_{+}$from $B D(\Omega)$ to $\left(L^{1}(S)\right)^{N}$ such that for every $\varphi \in(\mathscr{D}(\Omega))_{s}^{N^{2}}:$

$$
\begin{aligned}
\int_{\Omega_{-}} u_{i} \varphi_{i j, j} d x+\int_{\Omega_{-}} \varphi_{i j} d \varepsilon_{i j}(u) & =\int_{s}(\gamma-u)_{i} \varphi_{i j} v_{j} d s & & (\forall u \in B D(\Omega)) \\
\int_{\bar{\Omega}} u_{i} \varphi_{i j, j} d x+\int_{\bar{\Omega}} \varphi_{i j} d \varepsilon_{i j}(u) & =\int_{s}\left(\gamma_{+} u\right)_{i} \varphi_{i j} v_{j} d s & & (\forall u \in B D(\Omega))
\end{aligned}
$$

$u^{+}=\gamma_{+} u$ and $u^{-}=\gamma_{-} u$ are respectively the external and the internal trace of $u$ on $(S)$ (for $\Omega_{-}$). These traces are equal when $u$ is a regular function, but in the general case are different, due to a possible mass of $\varepsilon(u)$ on $(S)$.

\section{REFERENCES}

[1] H. Attouch and A. Damlamian, Strong solutions for parabolic variational inequalities, Nonlinear Anal. Th. Meth. Appl. 2, 329-353 (1978)

[2] H. Brezis, Opérateurs maximaux monotones et semi-groupes de contraction dans les espaces de Hilbert, North Holland, 1973

[3] M. Crandall and A. Pazy, Nonlinear evolution equations in Banach spaces, Israël J. Math. 11, 57-94 (1972)

[4] O. Debordes, Thèse de doctorat d'Etat, Marseille, 1977

[5] G. Duvaut and J. L. Lions, Les inéquations en mécanique et en physique, Dunod, Paris, 1972

[6] I. Ekeland and R. Temam, Analyse convexe et problemes variationnels, Dunod, Paris, 1974

[7] B. Halphen and Quoc Son Nguyen, Sur les matériaux standard généralisés, J. de Mécanique 14, 39-63 (1975)

[8] Ionescu-Tulcea, Topics in the theory of liftings, Springer-Verlag, Berlin, 1972

[9] C. Johnson, Existence theorems in plasticity, J. Math. Pures et Appl. 55, 431-44 (1976)

[10] C. Johnson and B. Mercier, Une méthode pour résoudre le probleme de ladaptation, C. R. Acad. Sc. Paris 283, 371-374 (1976)

[11] N. Kenmochi, The semi-discretization method and nonlinear time-dependent parabolic variational inequalities, Proc. Jap. Acad. 50, 714-717 (1975)

[12] W. Koiter, General theorems for elastoplastic solids, in Progress in solid mechanics, ed. Sneddon and Hill, North-Holland, 1964, 164-221

[13] R. Kohn, New estimates for deformations in terms of their strains, Ph.D. thesis, Princeton University, 1979

[14] A. Lichnewsky, Solutions généralisées du problème des surfaces minimales pour des données au bord non bornées, J. Math. Pures et Appl. 57, 231-254 (1978)

[15] J. L. Lions, Problemes aux limites dans les équations aux dérivées partielles, Presses de l'université de Montréal, 1965

[16] R. H. Martin, Generating an evolution system in a class of uniformly convex Banach spaces, J. Func. Anal. 11, 62-76 (1972)

[17] B. Mercier, Une méthode pour résoudre le problème des charges limites, J. de Mécaniquue, 16 (1977)

[18] M. Miranda, Distribuzioni aventi derivate misure, insiemi di perimetro localmente finito, Ann. di Pisa 18, 27-56 (1964)

[19] H. Matthies, Existence theorems in thermoplasticity, J. de Mécanique 18, 675-712 (1979)

[20] H. Matthies, G. Strang and E. Christiansen, The saddle point of a differential program, in Energy methods in finite element analysis, ed. Rodin, Zienkiewicz, Glowinski, Wiley, New York, 1979

[21] J. J. Moreau, On unilateral constraints, friction and plasticity, C.I.M.E. Bressanone, 1973

[22] J. J. Moreau, Application of convex analysis to the treatment of elasto-plastic systems, in Springer Lecture Notes in Math. No 503, ed. P. Germain and B. Nayroles, 1975

[23] J. J. Moreau, Evolution problem associated with a moving convex set in a Hilbert space, J. Diff. Eqs. 26, 347-374 (1977)

[24] J. J. Moreau, Champs et distributions de tenseurs déformation sur un ouvert de connexité quelconque, Sem. Anal. Convexe, Montpellier, 1976

[25] B. Nayroles, Essai de théorie fonctionnelle des structures rigides plastiques parfaites, J. de Mécanique 9, 491-506 (1970) 
[26] Quoc Son Nguyen, Matériau élastoplastique écrouissable, Ark. of Mech. 25, 695-702 (1973)

[27] G. Strang and R. Temam, Existence de solutions relaxées pour les équations de la plasticité: Etude d'un espace fonctionnel, C. R. Acad. Sc. Paris 287, 515-519 (1978)

[28] P. M. Suquet, Un espace fonctionnel pour les équations de la plasticité, C. R. Acad. Sc. Paris 286, 1129-1132 (1978).

[29] P. M. Suquet, Un espace fonctionnel pour les équations de la plasticité, Ann. Fac. Sc. Toulouse 1, 77-87 (1979)

[30] R. Temam, Mathematical problems in plasticity theory, in Proceedings of the symposium on complementary problems and variational inequalities, Erice (to appear)

[31] A. I. Volpert, The spaces BV and quasilinear equations, Mat. Sbornik 115, 225-267 (1967) 